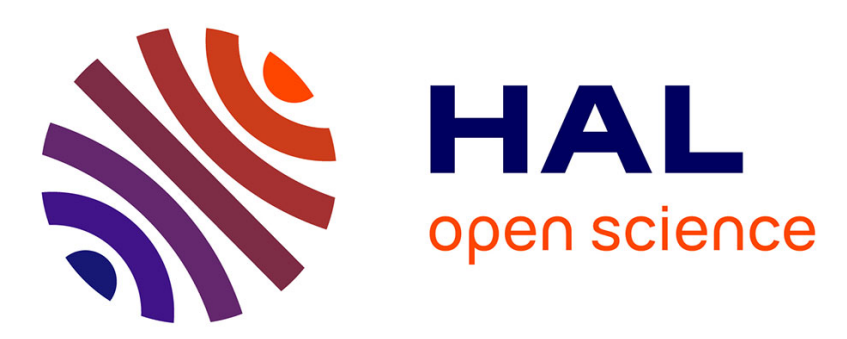

\title{
Comparative studies of the cage systems ThFe2Al10 and $\mathrm{UFe} 2 \mathrm{Al10}$
}

\author{
R. Troć, R. Wawryk, Z. Gajek, Mathieu Pasturel, M. Samsel-Czekala
}

\section{To cite this version:}

R. Troć, R. Wawryk, Z. Gajek, Mathieu Pasturel, M. Samsel-Czekala. Comparative studies of the cage systems ThFe2Al10 and UFe2Al10. Journal of Alloys and Compounds, 2017, 727, pp.1302-1313. 10.1016/j.jallcom.2017.08.225 . hal-01619415

\section{HAL Id: hal-01619415 \\ https://hal-univ-rennes1.archives-ouvertes.fr/hal-01619415}

Submitted on 7 Dec 2017

HAL is a multi-disciplinary open access archive for the deposit and dissemination of scientific research documents, whether they are published or not. The documents may come from teaching and research institutions in France or abroad, or from public or private research centers.
L'archive ouverte pluridisciplinaire HAL, est destinée au dépôt et à la diffusion de documents scientifiques de niveau recherche, publiés ou non, émanant des établissements d'enseignement et de recherche français ou étrangers, des laboratoires publics ou privés. 


\title{
Comparative studies of the cage systems $\mathrm{ThFe}_{2} \mathrm{Al}_{10}$ and $\mathrm{UFe}_{2} \mathrm{Al}_{10}$
}

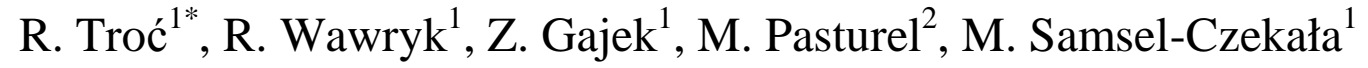 \\ ${ }^{1}$ W. Trzebiatowski Institute of Low Temperature and Structure Research, Polish Academy of Sciences, Okólna 2, \\ 50-422 Wroctaw, Poland \\ ${ }^{2}$ Institut des Sciences Chimiques de Rennes, Chimie du Solide et Matériaux, Université Rennes 1, UMR CNRS \\ 6226, Campus de Beaulieu, 263 av. Général Leclerc, 35042 Rennes Cedex, France
}

* Corresponding author: Tel.: +48 713954 206;

E-mail address: R.Troc@int.pan.wroc.pl (R. Troć)

\begin{abstract}
We have grown single crystals of $\mathrm{ThFe}_{2} \mathrm{Al}_{10}$ and used them for the refinement of its crystal structure. From the specific heat data, we determined the phonon components, that is, the Debye and Einstein modes, for Th- and Ubased isostructural aluminides. To approximate the Sommerfeld coefficients, we had to include the $T^{3} \ln T$ term characteristic of spin-fluctuations originating from the Fe-sublattice. The roughly estimated spin-fluctuation temperature is about $11 \mathrm{~K}$. ThFe ${ }_{2} \mathrm{Al}_{10}$ is a weakly temperature-dependent Pauli-paramagnet. However, at lower temperatures the ferromagnetic correlations are observed, which signals that the system drives to quantum criticality. The distinct minimum in electrical resistivity observed at about $20 \mathrm{~K}$ suggests a realization of a nonmagnetic two-channel Kondo-effect in $\mathrm{ThFe}_{2} \mathrm{Al}_{10}$, as discussed for ThAsSe [Phys. Rev. Lett. 94, 236603 (2005)], for example. The $\left[\rho(T)-\rho_{\min }\right]$ versus $T$ curves of $\mathrm{ThFe}_{2} \mathrm{Al}_{10}$, determined along the $a$ - and $c$-axes, were used to subtract the phonon contributions from the corresponding $\rho(T)_{a, c}$ of $\mathrm{UFe}_{2} \mathrm{Al}_{10}$. The resulting magnetic part of the average $\rho_{m}(T)_{\text {av }}$ curve was then analysed in terms of the influence of the crystal field effect on the transport properties. However, to obtain agreement with the experiment, we had to take into account another effect, namely the Kondo-like one. This kind of probe has been applied for the first time in the case of uranium compounds. Based on the magnetoresistivity, we have revealed the anisotropic low-frequency vibrations of the Th atom (located in its $\left[\mathrm{Al}_{16} \mathrm{Fe}_{4}\right]$ cage) interacting with the conduction band, the phenomenon revealed previously in the metallic $\mathrm{UB}_{12}$ [Phil. Mag. B 95, 2343 (2015)]. Furthermore, fully relativistic band structure calculations performed for $\mathrm{ThFe}_{2} \mathrm{Al}_{10}$ revealed its metallic-like character with a similarly large contribution of the $\mathrm{Fe} 3 d$ electrons at the Fermi level as predicted previously for its $5 f$-electron analogue $\mathrm{UFe}_{2} \mathrm{Al}_{10}$. In addition, there are substantial differences between their Fermi surfaces.
\end{abstract}

\section{Keywords:}

Actinide alloys and compounds; Heat capacity; Magnetoresistance; Electron-phonon interactions; Kondo effect; Electronic band structure 


\section{Introduction}

The U-Fe-Al system exhibits several ternary phases [1]. Among the most intensively investigated phases are those with the generalized formula of $\mathrm{UFe}_{x} \mathrm{Al}_{12-\mathrm{x}}$, which possess a wide $\mathrm{Fe}$-content range for $3.8<\mathrm{x}<5.8$. In this composition range, four magnetic phases have been identified mainly due to the canted antiferromagnetic order of the Fe-sublattice, while the U-sublattice becomes ferromagnetically ordered in all four regions [2]. All these phases crystallize in the tetragonal $\mathrm{ThMn}_{12^{-}}$ type structure. Interestingly, the stoichiometric phase considered here with $\mathrm{x}=2, A n \mathrm{Fe}_{2} \mathrm{Al}_{10}$ (where $A n$ $=\mathrm{Th}$ and $\mathrm{U}$ ), adopts a different structure of the orthorhombic $\mathrm{YbFe}_{2} \mathrm{Al}_{10}$-type [3]. Zenou et al. [4], based on the total energy calculations using the full potential band structure method with linearized augmented plane waves (FP-LAPW) with the spin-orbit (SO) coupling correction, explained why the $\mathrm{UFe}_{2} \mathrm{Al}_{10}$ compound has a different crystal structure from the $\mathrm{ThMn}_{12}$-type one. Of course, their arguments also concerned the Th-based aluminides with this composition. Generally, the $M T_{2} \mathrm{Al}_{10}$ ternaries (where $M$ is a guest atom and $T$ is a transition metal) belong to a cage family of compounds like those with the compositions $M T_{4} X_{12}(X=\mathrm{P}, \mathrm{As}$ and $\mathrm{Sb})$ - the so-called skutterudites - or $M T_{2} Z_{20}(Z$ $=\mathrm{Al}, \mathrm{Ga}$, or In). The characteristic feature of this family of ternaries is that they form a rigid covalently bonded cage, which is also due to a donation of electrons from a given accommodated $M$ atom. All members of this cage-like family of ternaries have recently attracted much attention because they exhibit a large diversity of physical properties due to a variety of guest atoms and interframework interactions.

The previously investigated polycrystalline $\mathrm{UFe}_{2} \mathrm{Al}_{10}$, despite showing modified CurieWeiss behaviour, appeared to be a non-magnetically-ordered compound down to the lowest temperatures, as evidenced by magnetization, magnetic susceptibility, and neutron-diffraction measurements [5-7]. Among the family of isostructural rare-earth ternaries, $R \mathrm{Fe}_{2} \mathrm{Al}_{10}$, similar magnetic properties were found for the light representatives, that is, when $R=\mathrm{Ce}$ and Pr. Interestingly, such a compound based on Ce was classified as a mixed-valence type and also as a Kondo semiconductor $[8,9]$. It should be noted here that the Ce-based compounds containing Ru or Os instead of $\mathrm{Fe}$ have recently attracted great interest due to their antiferromagnetic ordering with unexpected very high Néel temperatures of about $30 \mathrm{~K}$ [Ref. 10 and references therein]. Indeed, taking into account the substantial distance $\mathrm{d}_{\mathrm{Ce}-\mathrm{Ce}}$ of $5.2 \AA$ in these ternaries, one could only expect a value of $T_{N}$ one order of magnitude lower, such as that for $\mathrm{NdFe}_{2} \mathrm{Al}_{10}(3.9 \mathrm{~K})$ [11], for example. This large difference in values of $T_{N}$ was interpreted by the authors of Ref. [10] as originating from a twodimensional strong $c-f$ hybridization between conduction and $4 f$ electrons in the $(a c)$-plane, which, in consequence, causes a charge-density wave (CDW) instability in $\mathrm{CeRu}_{2} \mathrm{Al}_{10}$ and $\mathrm{CeOs}_{2} \mathrm{Al}_{10}$. Therefore, this instability is, according to these authors, responsible for inducing an untypical antiferromagnetic state in these two ternaries, where a CDW energy gap is opened along the $b$ axis, that is, where the $c-f$ hybridization is the weakest one. All the remaining Fe-based heavier representatives with $R=\mathrm{Sm}$ and $\mathrm{Gd}-\mathrm{Tm}$ have also been reported to be magnetically ordered, with the highest transition temperature of $20 \mathrm{~K}$ for $\mathrm{GdFe}_{2} \mathrm{Al}_{10}$ [8]. On the other hand, the iron-containing compounds with $R=\mathrm{Y}$ and La were found to be Pauli-like paramagnets [8]. Later works by Strydom et al. $[12,13]$ pointed out a tendency of $\mathrm{YFe}_{2} \mathrm{Al}_{10}$ toward a low-temperature ferromagnetic instability driving this compound into ferromagnetic quantum criticality of Fe moments leading to $\mathrm{T} \rightarrow 0$ phase transition. The latter properties yield arguments that the iron atoms in these two intermetallics do not carry a magnetic moment. To verify this claim, in this paper we present magnetic and both electrical and thermal transport property studies of $\mathrm{ThFe}_{2} \mathrm{Al}_{10}$. Knowledge of these properties allows a comparison to be drawn with those of the U-containing isomorphic compound, $\mathrm{UFe}_{2} \mathrm{Al}_{10}$, whose physical properties have recently been investigated in detail using single-crystalline materials $[14,15]$.

In our previous work [15], we used the measured specific heat data only to extract the Schottky effect in $\mathrm{UFe}_{2} \mathrm{Al}_{10}$ following the inferred scheme of crystal field (CF) levels determined there. Their splitting could satisfactorily explain the large magnetic anisotropy acting in this CurieWeiss paramagnet even down to $0.3 \mathrm{~K}$. Thus, in this work we compare the results of this U-based aluminide with those obtained for a $\mathrm{ThFe}_{2} \mathrm{Al}_{10}$ single crystal and carry out a full analysis of the data of the specific heat. Moreover, we present the results of band structure calculations for $\mathrm{ThFe}_{2} \mathrm{Al}_{10}$, which can be regarded as a reference compound for $\mathrm{UFe}_{2} \mathrm{Al}_{10}$ because of the lack of $5 f$-electrons in its $[\mathrm{Rn}] 6 d^{2} 7 s^{2}$ atomic configuration. For example, the topology of the Fermi surface (FS) of this thorium 
compound is compared to that in the corresponding uranium compound as well as those in $\mathrm{URu}_{2} \mathrm{Al}_{10}$ and $\mathrm{CeRu}_{2} \mathrm{Al}_{10}$, which were reported earlier [16].

\section{Experimental and calculation details}

\subsection{Bulk measurements}

$\mathrm{ThFe}_{2} \mathrm{Al}_{10}$ and $\mathrm{UFe}_{2} \mathrm{Al}_{10}$ single crystals were prepared by the molten aluminium flux technique, as described in Ref. [15]. Their chemical composition and crystallographic quality and orientation were verified by both scanning electron microscopy coupled to energy dispersive spectroscopy (SEM-EDS) and $\mathrm{x}$-ray diffraction (XRD) techniques. No impurity phase inclusion was observed by either technique. Moreover, the XRD examination of powdered single crystals shows up very sharp reflections and the lack of any foreign phases. It should be also noted that in general it is very difficult to obtain high quality crystals of thorium compounds.

The diffraction intensities were collected at room temperature (RT) on a Nonius Kappa CCD four-circle diffractometer equipped with a bidimensional CCD detector and using monochromatized Mo $K_{\alpha}$ radiation $(\lambda=0.71073 \AA)$. The integration and reduction of redundant reflections of the different data sets as well as the cell refinements were performed using SADABS software [17]. Structural models were determined by direct methods using SIR-97 [18]. All the structural refinements and Fourier syntheses were carried out with the help of SHELXL-97 [19]. The atomic positions were standardized using STRUCTURE TIDY [20]. The lattice parameters of $\mathrm{ThFe}_{2} \mathrm{Al}_{10}$ were collected in Table 1. Most of the crystals presented the $a$-axis perpendicular to the plate base.

The magnetic susceptibility between 2 and $300 \mathrm{~K}$ and magnetization at low temperatures of up to $5 \mathrm{~T}$ were measured using a Quantum Design MPMS-5 magnetometer. The specific heat of the single-crystalline sample of $\mathrm{ThFe}_{2} \mathrm{Al}_{10}$ with a mass of $29.2 \mathrm{mg}$ was measured by the thermal relaxation method in the temperature range of 2-300 K using a commercial Quantum Design PPMS platform. The samples were glued to the holder using Apiezon $\mathrm{N}$ vacuum grease addenda, whose specific heat was measured earlier.

Similarly to the electrical resistivity measurements for the $\mathrm{UFe}_{2} \mathrm{Al}_{10}$ single crystals [15], the $\mathrm{ThFe}_{2} \mathrm{Al}_{10}$ electrical resistivity measurements were performed on samples in the form of bar-shaped specimens, which we cut from the irregular plate-like single crystals, with dimensions of about $1.5 \times$ $0.5 \times 0.4 \mathrm{~mm}^{3}$ along the $a$ direction and $1.0 \times 0.5 \times 0.4 \mathrm{~mm}^{3}$ along the $c$ direction. The electrical contacts (four points) were made by the electrochemical deposition of $\mathrm{Cu}$, and finally silver thin wires were glued by using a silver paste. The measurements were carried out in a ${ }^{3} \mathrm{He}$ cryostat in the temperature range of $0.3-300 \mathrm{~K}$ using an $\mathrm{AC}$ method. The magnetoresistivity was measured on the same samples in an applied field of $9 \mathrm{~T}$. After measurements in the magnetic field we repeated the measurements without applying the field in order to be sure of good electric contacts. In turn, a homemade set-up [21] was used for measurements of the thermoelectric power of $\mathrm{ThFe}_{2} \mathrm{Al}_{10}$ at temperatures from 0.4 to $300 \mathrm{~K}$, in the same way as for the U-based aluminide [15]. The frontal surfaces of the same samples along the $a$ and $c$ directions were wetted with a liquid In-Ga alloy in order to improve the thermal and electrical contacts with chamber plates.

\subsection{Band structure calculations}

The band structure of $\mathrm{ThFe}_{2} \mathrm{Al}_{10}$ has been calculated, like that for $\mathrm{UFe}_{2} \mathrm{Al}_{10}$ [15], employing the fully relativistic version of the full-potential local-orbital (FPLO) method [22]. In this method, all electrons are treated by the four-component Kohn-Sham-Dirac equation, implicitly taking into account all relativistic effects including SO coupling, which is solved in the self-consistent way. The PerdewWang form [23] of the local density approximation (LDA) of the exchange-correlation functional was assumed. Two sets of experimental (XRD) lattice parameters and atomic positions in the orthorhombic unit cell (u.c.) of the $\mathrm{YbFe}_{2} \mathrm{Al}_{10}$-type ( $\mathrm{Cmcm}$, space group no. 63) were considered. The first set (set. 1) consisted of the crystallographic data determined for our sample, which are presented in Table 1 , and the other set (set. 2) was taken from Ref. [24]. In addition, for set 1 the self-consistent spinpolarised total energy calculations were performed by employing both local spin density approximation (LSDA) and generalized gradient approximation (GGA) [25], assuming a 
ferromagnetic state, but the non-magnetically-ordered state was obtained again. In the version of the FPLO code used here, the valence-basis sets have been automatically selected by the internal procedure. The atomic-orbital-based FPLO code yielded the Mulliken decomposition of charges, which is somewhat dependent on the orbital used. The $12 \times 12 \times 12$ size of the selected $\boldsymbol{k}$-point meshes corresponded to 301 points in the irreducible wedge of the Brillouin zone (BZ). The band structures, total and partial densities of states (DOS), and FSs were computed. The partial DOSs were obtained for different atomic sites or electron orbitals and plotted per formula unit (f.u.).

\section{Results and discussion}

\subsection{Crystal and Electronic Structures}

\subsubsection{Crystal Structure Refinement}

The structural characterization of the ternary aluminide $\mathrm{ThFe}_{2} \mathrm{Al}_{10}$ was reported by Meshi et al. in 2005 from Rietveld refinement of the powder XRD pattern [24]. They revealed that this phase crystallizes like $\mathrm{UFe}_{2} \mathrm{Al}_{10}$ into the orthorhombic (space-group Cmcm) structure of $\mathrm{YbFe}_{2} \mathrm{Al}_{10}$-type with unit cell parameters $a=8.9806, b=10.2520$, and $c=9.0882 \AA$. This crystal structure type was detailed by Niemann and Jeitschko [26] about two decades ago. In this structure, the $\mathrm{Yb}$ atoms are located at the $4 c$ position, $\mathrm{Fe}$ atoms at $8 d$, and $\mathrm{Al}$ atoms at the $2 \times 8 g, 2 \times 8 f$, and $1 \times 8 e$ positions. The uranium atom has a coordination number $(\mathrm{CN})$ of $20(4 \mathrm{Fe}+16 \mathrm{Al})$, while the iron atom forms in its coordination a distorted icosahedron made of $2 \mathrm{U}$ and $10 \mathrm{Al}$ atoms, as shown in Figs. 1(a) and (b), respectively. It should be noted that an asymmetric Fe-Al cage is responsible for strong anisotropy of the physical properties in $\mathrm{UT}_{2} \mathrm{Al}_{10}$ aluminides $(T=\mathrm{Fe}, \mathrm{Ru}$, and $\mathrm{Os})$ [14, 15]. Moreover, this cage also gives rise to anisotropic $c-f$ hybridization in these types of Ce-ternaries (see e.g. Refs. 9, 10). In turn, the five different $\mathrm{Al}$ atoms coordinate in irregular polyhedra consisting of $\mathrm{U}, \mathrm{Fe}$, or $\mathrm{Al}$ atoms with $\mathrm{CN}$ ranging from 12 to 14 . It is interesting to note that a similar coordination around the $T$ atom $(T=\mathrm{Ti}, \mathrm{V}$, and $\mathrm{Cr}$ ) is formed in the case of $\mathrm{Th}_{2} \mathrm{Al}_{20}$ series, crystallizing in the cubic $\mathrm{CeCr}_{2} \mathrm{Al}_{20}$ type of structure [27]. In contrast to $\mathrm{ThFe}_{2} \mathrm{Al}_{10}$, the coordination sphere there is a regular icosahedron $T$ - $\mathrm{Al}_{12}$, which is, however, unstable when $T=\mathrm{Fe}$.

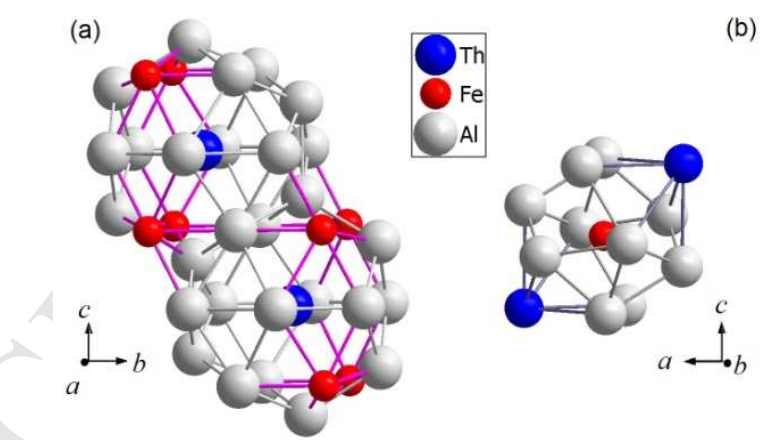

Fig. 1. (a) Two jointed Th- $(16 \mathrm{Al}+4 \mathrm{Fe})$ caged polyhedra. Note that Th atoms form a zigzag chain prolonged along the $c$ axis. (b). The fundamental polyhedron $[\mathrm{Fe}-(10 \mathrm{Al}+2 \mathrm{Th})]$ in the $\mathrm{YbFe}_{2} \mathrm{Al}_{10}$-type structure. The latter polyhedra are connected in the $a c$ plane and in such a two-dimensional system form crevices, in which the Th atoms are located. They possibly give rise to anisotropic rattling vibrations inside these crevices.

The corresponding refined atomic positions and isotropic thermal displacement factors of the Th-bearing phase are given in [24], but in order to gain higher precision for electronic structure calculations, we performed single-crystal XRD experiments. Details on the data collection and structure refinements can be found in Table 1, while atomic positions and the equivalent isotropic displacement parameters are given in Table 2. The seven atomic positions of the $\mathrm{YbFe}_{2} \mathrm{Al}_{10}$-type structure are confirmed and all of them are fully occupied by a single kind of atom. The relevant interatomic distances are listed in Table 3.

Despite the cage-like type of structure of $\mathrm{UFe}_{2} \mathrm{Al}_{10}$ and the U-ligand distances exceeding the sum of the metallic radii of the elements [6], an increase of the u.c. volume of approximately $3.3 \%$ is observed when $\mathrm{U}$ is substituted by Th. 
Table 1 Crystallographic data and structure refinement for $\mathrm{ThFe}_{2} \mathrm{Al}_{10}$.

\begin{tabular}{l|l}
\hline Empirical formula & $\mathrm{ThFe}_{2} \mathrm{Al}_{10}$ \\
Formula weight $\left(\mathrm{g} \mathrm{mol}^{-1}\right)$ & 613.54 \\
Structure-type & $\mathrm{CeFe}_{2} \mathrm{Al}_{10}$ \\
Space group & $\begin{array}{l}C m c m\left(\mathrm{n}^{\circ} 63\right) \\
a=9.017(1)\end{array}$ \\
& $b=10.293(1)$ \\
Unit cell parameters $(\AA)$ & $8=9.116(1)$ \\
Unit cell volume $\left(\AA^{3}\right)$ & $846.1(2)$ \\
$\mathrm{Z} /$ calculated density $\left(\mathrm{g} \mathrm{cm}^{-3}\right)$ & $4 / 4.817$ \\
Absorption coefficient $\left(\mathrm{mm}^{-1}\right)$ & 21.875 \\
Crystal colour and habit & metallic lustre \\
Theta range & $3.74^{\circ}$ to $41.99^{\circ}$ \\
& $-13 \leq \mathrm{h} \leq 16$ \\
Limiting indices & $-17 \leq \mathrm{k} \leq 19$ \\
& $-17 \leq 1 \leq 17$ \\
Collected/unique reflections & $11069 / 1608$ \\
$\mathrm{R}($ int $)$ & 0.074 \\
Data / restraints / parameters & $1608 / 0 / 41$ \\
Goodness of fit on F & 0.999 \\
R indices [I>2 $\sigma(\mathrm{I})]$ & $\mathrm{R} 1=0.0298$ \\
Extinction coefficient & $\mathrm{wR} 2=0.0513$ \\
Largest difference peak and & $0.0085(3)$ \\
hole (e $\left.\AA^{-3}\right)$ & $6.375 /-3.350$ \\
\hline
\end{tabular}

This increase can be understood by considering the metallic radius of these actinides $\left(\mathrm{r}_{\mathrm{Th}}=\right.$ $1.798 \AA$ versus $\left.r_{U}=1.56 \AA[28]\right)$ and the thorium-to-aluminium distances $\left(\mathrm{r}_{\mathrm{Al}}=1.432 \AA\right)$ : in $\mathrm{ThFe}_{2} \mathrm{Al}_{10}$, the shortest $\mathrm{Th}-\mathrm{Al}$ distances are slightly smaller than the sum of the metallic radii of the elements and correspond well to those encountered in, for example, most of the Th-Al binaries (around $3.2 \AA$ [29]). The cage-like character of this Th-based aluminide is thus expected to be considerably weakened compared to the U-based one. For the latter, we have to do rather with a uranium ion $\mathrm{U}^{4+}$, and hence its chemical bonding with the cage is much stronger than that of Th atoms, which have a more metallic character.

Table 2 Atomic parameters and equivalent displacement parameters for $\mathrm{ThFe}_{2} \mathrm{Al}_{10}$.

\begin{tabular}{llllll}
\hline Atoms & $\begin{array}{l}\text { Wyckoff } \\
\text { position }\end{array}$ & $\mathrm{x}$ & $\mathrm{y}$ & $\mathrm{z}$ & $U_{\mathrm{eq}}\left(\AA^{2}\right)$ \\
\hline Th1 & $4 c$ & 0 & $0.3732(1)$ & $1 / 4$ & $0.008(1)$ \\
$\mathrm{Fe} 1$ & $8 d$ & $1 / 4$ & $1 / 4$ & 0 & $0.007(1)$ \\
$\mathrm{Al} 1$ & $8 g$ & $0.2280(1)$ & $0.1360(1)$ & $1 / 4$ & $0.008(1)$ \\
$\mathrm{Al} 2$ & $8 g$ & $0.3519(1)$ & $0.3707(1)$ & $1 / 4$ & $0.008(1)$ \\
$\mathrm{Al} 3$ & $8 f$ & 0 & $0.1228(1)$ & $0.0488(1)$ & $0.009(1)$ \\
$\mathrm{Al} 4$ & $8 f$ & 0 & $0.6600(1)$ & $0.0997(1)$ & $0.008(1)$ \\
$\mathrm{Al} 5$ & $8 e$ & $0.2722(1)$ & 0 & 0 & $0.006(1)$ \\
\hline
\end{tabular}

Table 3 Selected interatomic distances $(\AA)$ for $\mathrm{ThFe}_{2} \mathrm{Al}_{10}$.

\begin{tabular}{rlrr} 
Th $-2 \mathrm{Al} 3$ & $3.164(2)$ & $\mathrm{Fe}-2 \mathrm{All}$ & $2.5710(6)$ \\
$-2 \mathrm{Al} 2$ & $3.173(2)$ & $-2 \mathrm{Al} 5$ & $2.5810(3)$ \\
$-2 \mathrm{Al1}$ & $3.192(2)$ & $-2 \mathrm{Al} 4$ & $2.6010(6)$ \\
$-2 \mathrm{Al} 4$ & $3.206(2)$ & $-2 \mathrm{Al} 3$ & $2.6446(7)$ \\
$-2 \mathrm{Al} 4$ & $3.254(2)$ & $-2 \mathrm{Al} 2$ & $2.7533(7)$ \\
$-4 \mathrm{Al} 5$ & $3.3343(8)$ & & \\
$-4 \mathrm{Fe} 1$ & $3.4474(2)$ & & \\
$-2 \mathrm{Al1}$ & $3.6212(3)$ & & \\
\hline
\end{tabular}




\subsubsection{Electronic structure}

For $\mathrm{ThFe}_{2} \mathrm{Al}_{10}$, our fully relativistic band structure calculations for the considered two sets of somewhat different lattice parameters (by about $0.040 \AA$ ) and very similar (after transformation to the same coordinate system) atomic positions (within 0.002) yielded a substantially lower (by $50 \mathrm{mHa}$ $\sim 1.36 \mathrm{eV} /$ f.u.) total energy for set 1 , corresponding to our refinement, than for set 2 . This indicates that our estimation is better than the one reported in Ref. [24]. For both sets of data, the calculated bandplots and DOS, presented in Figs. 2 (a) and (b), respectively, and the related FSs (not shown) are also very different. Therefore, we limit further discussion to the data of set 1.

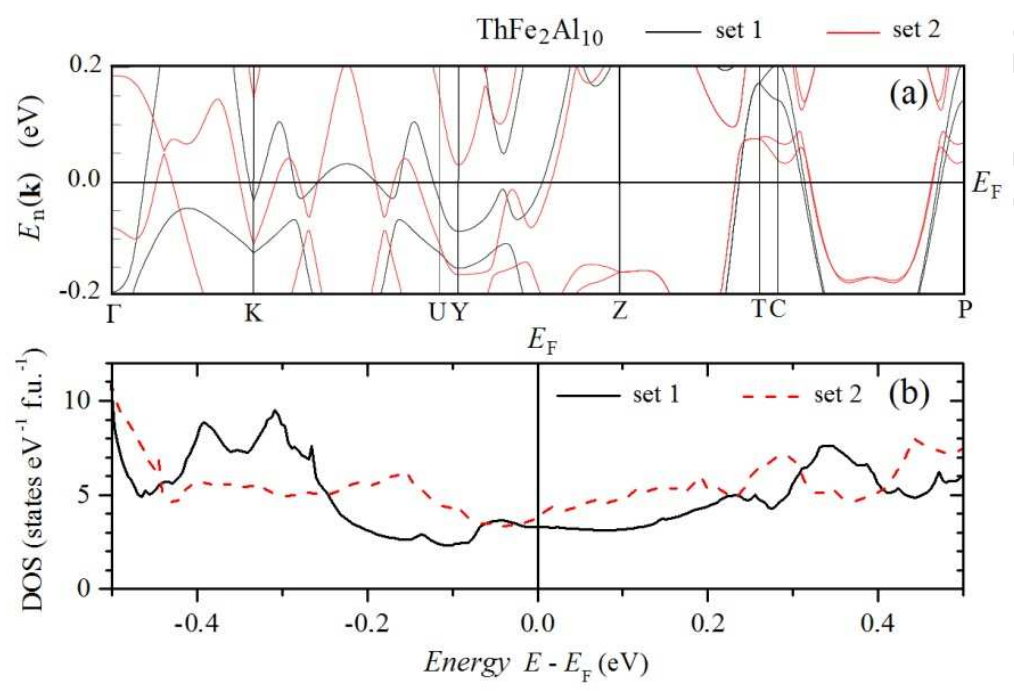

Fig. 2. (a) Fully relativistic (LDA) bandplots of $\mathrm{ThFe}_{2} \mathrm{Al}_{10}$, calculated for two different sets of crystallographic data (sets 1 and 2). (b) The corresponding total DOS in the vicinity of $E_{\mathrm{F}}$.

The fully relativistic total and partial DOS of $\mathrm{ThFe}_{2} \mathrm{Al}_{10}$ (within LDA) are displayed in Fig. 3 and the related FS is visualized in Fig. 4. As seen in Fig. 3, all the constituent atoms of $\mathrm{ThFe}_{2} \mathrm{Al}_{10}$, forming a cage-type crystal structure, contribute to the DOS at the Fermi level $\left(E_{\mathrm{F}}\right)$ as in the case of isostructural counterparts $\mathrm{UFe}_{2} \mathrm{Al}_{10}[15]$ and $(\mathrm{U} ; \mathrm{Ce}) \mathrm{Ru}_{2} \mathrm{Al}_{10}$ [16]. However, the valley of DOS cuts $E_{\mathrm{F}}$, yielding a not too large value of the Sommerfeld coefficient $\gamma_{6}=7.8 \mathrm{~mJ} \mathrm{~mol}^{-1} \mathrm{~K}^{-2}$, which is typical of normal metallic systems, while our experimentally estimated values are rather high, indicating the presence of electron-electron and electron-phonon correlations enhanced by at least a factor of two; for details see subsection 3.2.1 below. The DOS around $E_{\mathrm{F}}$ is dominated by almost equal contributions from the $\mathrm{Fe} 3 d$ and $\mathrm{Al} 3$ spd electrons, with the latter coming from all five atomic sites in the u.c. Also in $\mathrm{UFe}_{2} \mathrm{Al}_{10}$ [15], the corresponding $\mathrm{Fe}$ and $\mathrm{Al}$ contributions were equal at the Fermi level and had comparable values to those in the Th-based system. However, in the U-bearing compound the U $5 f$ contributions were three times higher than the $\mathrm{Fe}$ and $\mathrm{Al}$ ones.

In turn, the thorium contribution from the hybridized Th $6 d$ and $5 f$ states yields a long tail originating mainly from the Th $6 d$ electrons far below $E_{\mathrm{F}}$ (see the hatched area in Fig. 3), whereas above $E_{\mathrm{F}}$, unoccupied $5 f$ electrons start to predominate within the Th contribution. The $\mathrm{Al} 3 s p d$ states coming from five non-equivalent sites of $\mathrm{Al}$ atoms form a wide structure in the whole region of the valence and conduction bands. Meantime, the $\mathrm{Fe} 3 d$ states create a prominent multipeak structure with a maximum at around $-1.5 \mathrm{eV}$. It is interesting to note that the DOS coming from $\mathrm{Fe}$ and all the $\mathrm{Al}$ atomic positions in $\mathrm{ThFe}_{2} \mathrm{Al}_{10}$ resemble those of $\mathrm{UFe}_{2} \mathrm{Al}_{10}$ [15] in the whole energy range of the figures, which means that the band structure originating from the assembly $\left[\mathrm{Fe}_{2} \mathrm{Al}_{10}\right]$ is almost the same in both these aluminides.

Very recently, Uziel et al. [27] performed total energy calculations using a scalar relativistic FP-LAPW method within GGA as implemented in the Wien2k code for the cubic and orthorhombic phases of $\mathrm{Th}_{2} \mathrm{Al}_{\mathrm{x}}(\mathrm{x}=20$ and 10, respectively) considering different atoms of transition metals $T$. 


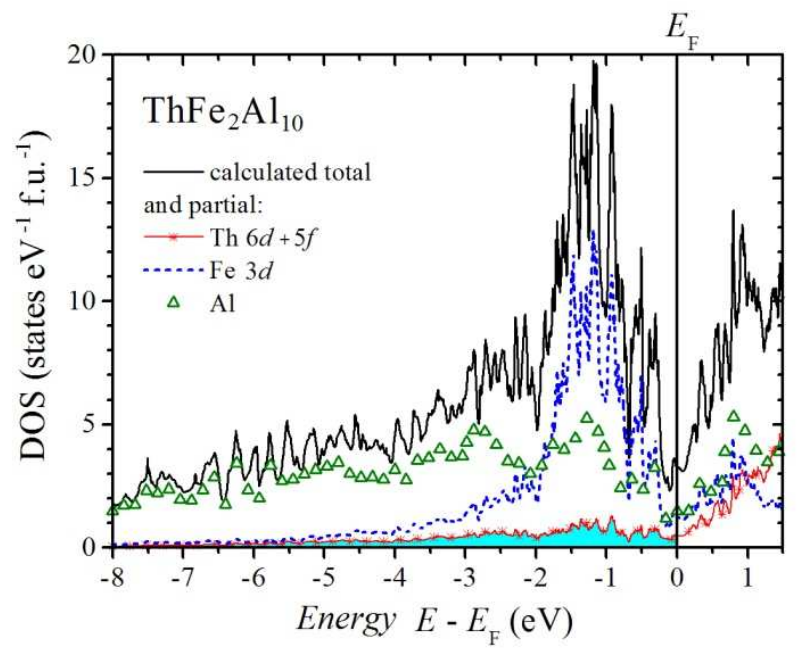

Fig. 3. Calculated fully relativistic (LDA) total and partial DOS of $\mathrm{ThFe}_{2} \mathrm{Al}_{10}$. The cyan (hatched) area denotes primarily occupied Th $6 d$ states.

Fermi surface of $\mathrm{ThFe}_{2} \mathrm{Al}_{10}$
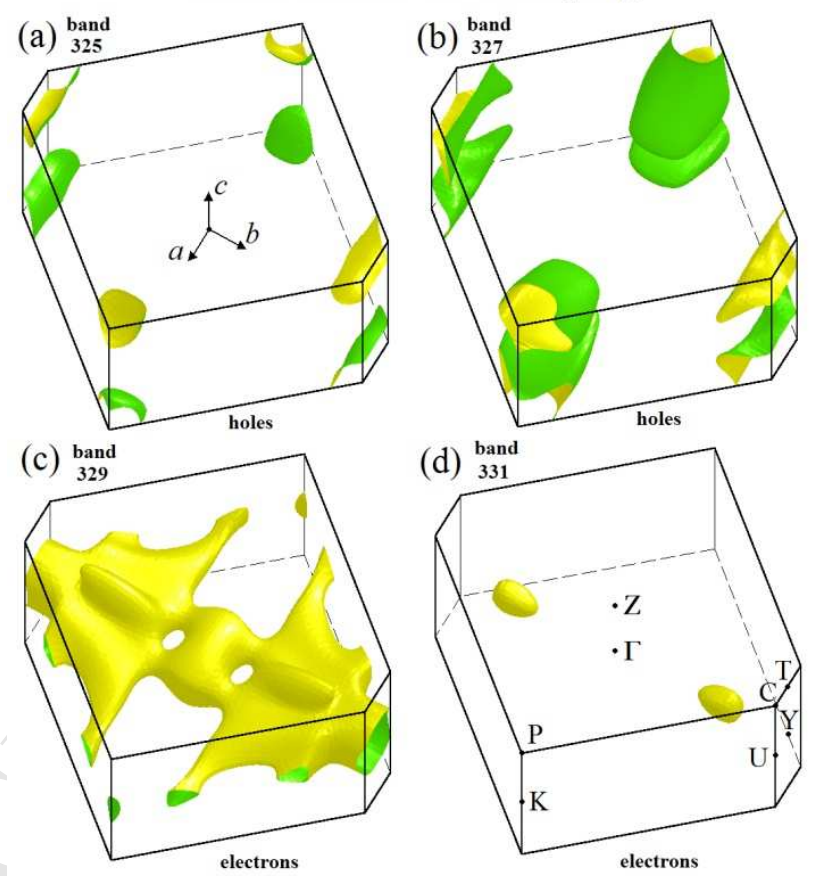

Fig. 4. Calculated fully relativistic (LDA) FS sheets of $\mathrm{ThFe}_{2} \mathrm{Al}_{10}$ originating from four Kramers doubledegenerate bands (nos. 325, 327, 329, 331), drawn separately within the orthorhombic BZ boundaries. Dark (green) and light (yellow) colors reflects the inside (electrons) and outside (holes) of FS, respectively.

However, they did not show any electronic structure results. Based on the total energy difference, they proved only that the orthorhombic Cmcm crystal structure is stable for $\mathrm{ThFe}_{2} \mathrm{Al}_{10}$ as for $\mathrm{ThFe}_{2} \mathrm{Al}_{20}$. These findings agree well with the experimental data. Furthermore, for the orthorhombic $\mathrm{ThFe}_{2} \mathrm{Al}_{10}$ phase, the authors also predicted a zero total magnetic moment on Fe atoms, while for the unstable cubic phase they found a small Fe-moment, which, according to the authors, provides another proof of instability of the latter phase. Also, our fully relativistic results, as mentioned in subsection 2.2, based on somewhat different from those given in [27] crystallographic data (set 1), have confirmed that the Fe atoms cannot become magnetically ordered even at $T=0 \mathrm{~K}$. As shown below, the latter result may contradict the experimental data.

Finally, as can be seen in Fig. 4, the computed (LDA) FS of $\mathrm{ThFe}_{2} \mathrm{Al}_{10}$ consists of as many as four sheets originating from Kramers double-degenerate bands: the 325th, 327th, 329th, and 331th 
bands. The sheets coming from two lower bands contain three-dimensional hole-like small closed pockets. They are located in the corners of the BZ, whereas the other two sheets possess electron-like character with large anisotropic (i.e. the $c$ direction versus the $a b$ plane - hybridization anisotropy) and flattened open structure (from the 329th band) and small closed pockets (from the 331th band). Such an FS suggests a more distinct metallic behaviour of Th-based aluminide compared to $\mathrm{UFe}_{2} \mathrm{Al}_{10}$ [15], which exists in only two bands and yields only closed pockets. Interestingly, the FS sheets of $\mathrm{ThFe}_{2} \mathrm{Al}_{10}$ are very similar to those of isostructural and isoelectronic $\mathrm{CeRu}_{2} \mathrm{Al}_{10}$ [16] except for the lack of the fourth small sheet in the latter system, although their DOS around $E_{\mathrm{F}}$ are quite different. Such a relation of FSs is analogous to that existing between, for example, $\mathrm{ThRhIn}_{5}$ and $\mathrm{CeCoIn}_{5}$ systems [30].

\subsection{Bulk properties}

\subsubsection{Thermal properties}

The specific heat data, $C_{\mathrm{p}}(T)$, of both $\mathrm{UFe}_{2} \mathrm{Al}_{10}$ and the reference compound $\mathrm{ThFe}_{2} \mathrm{Al}_{10}$ have been remeasured and the results were only negligibly different from the previous ones [15]. As demonstrated before, one observes the lack of any type of anomaly associated with a magnetic ordering in the whole temperature range measured. In Fig. 5, we have plotted only the data of $C_{\mathrm{p}}(T)$ for the Th-bearing compound, and these data are shown here as an example. They have been deconvoluted into three constituents: $C_{\mathrm{D}}, C_{\mathrm{E}}$, and $C_{\mathrm{e}}$, that is, into the Debye, Einstein, and electronic parts, respectively. The same procedure has been applied to the $C_{\mathrm{p}}(T)$ data of the U-bearing compound (not shown here).

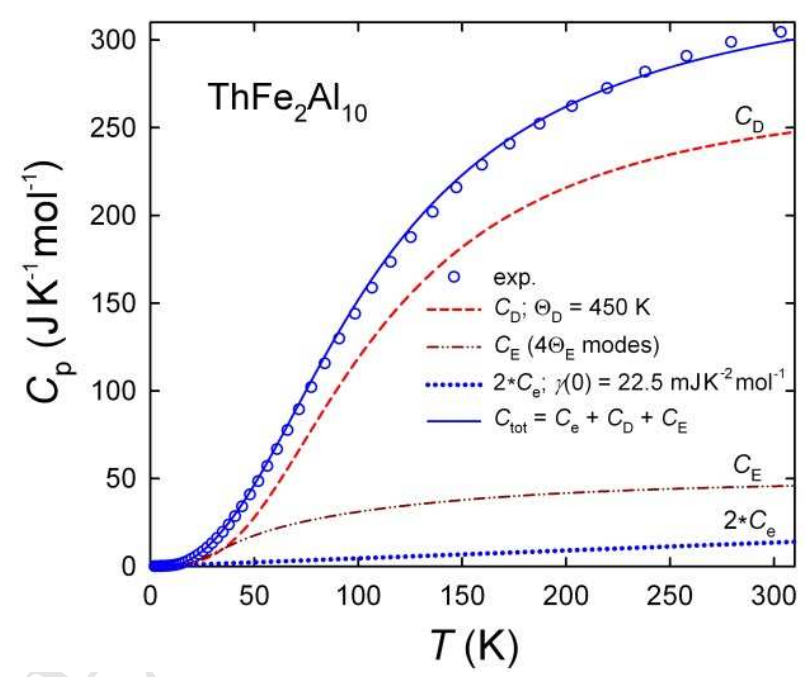

Fig. 5. The temperature variation of the specific heat $C_{\mathrm{p}}$ of $\mathrm{ThFe}_{2} \mathrm{Al}_{10}$ deconvoluted into three different contributions: Debye $C_{\mathrm{D}}$, Einstein $C_{\mathrm{E}}$ and electronic $C_{\mathrm{e}}$ constituents.

The electronic specific heat constants $\gamma(0)$ and the Debye temperatures $\Theta_{\mathrm{D}}$ were also recalculated, this time paying greater attention to the slight curvature and upward deviation from the approximately linear behaviour of the $C_{\mathrm{p}} / T$ versus $T^{2}$ function observed at low temperatures for both compounds, as displayed in Fig. 6. In the previous paper [15], we omitted a detailed analysis of this issue and now it has been possible to treat it much carefully owing to the repeated low-temperature measurements. Note that this upturn deviation is seen more distinctly for the Th- compound. We think that this feature might be evidence of the presence of the spin fluctuation behaviour at the Fe atom sites. Taking into account this feature, we have fitted the specific heat results for both studied compounds by applying Eq. (1) according to the theoretical prediction [31]:

$$
C_{\mathrm{p}} / T=A+B T^{2}+D T^{2} \ln T
$$

where the last term describes only the presence of spin fluctuation effects. At $T=0 \mathrm{~K}$, Eq. (1) yields the fitting values $A \equiv \gamma(0)_{\text {fit }} \approx 28.5(5)$ and $22.5(5) \mathrm{mJ} \mathrm{K}^{-2} \mathrm{~mol}^{-1}$ for the $\mathrm{U}$ - and Th-based aluminides, respectively. It should be realized that in the case of the latter aluminide, the proper value of this coefficient can be obtained by extrapolation of the $C_{\mathrm{p}} / T$ experimental data to $T=0$ and then $\gamma(0)_{\text {extr }}=$ 
25.0(1) $\mathrm{mJ} \mathrm{K}^{-2} \mathrm{~mol}^{-1}$. However, there is practically no difference between $\gamma(0)_{\mathrm{fit}}$ and $\gamma(0)_{\mathrm{extr}}$ in the case of U-based aluminide. The values of the above coefficients obtained in a different manner as well as the constant parameters $B \equiv \beta^{*}$ and $D \equiv \delta$ are gathered in Table 4 .

Table 4 The thermal properties of $\mathrm{ThFe}_{2} \mathrm{Al}_{10}$ compared to those of isostructural $\mathrm{UFe}_{2} \mathrm{Al}_{10}$.

\begin{tabular}{|c|c|c|c|c|c|c|c|c|}
\hline Compound & 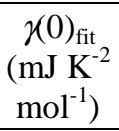 & $\begin{array}{c}\beta^{*} \\
\left(\mathrm{~mJ} \mathrm{~K}^{-4}\right. \\
\left.\mathrm{mol}^{-1}\right)\end{array}$ & $\begin{array}{c}\delta \\
\left(\mathrm{mJ} \mathrm{K}^{-4}\right. \\
\left.\mathrm{mol}^{-1}\right)\end{array}$ & $\begin{array}{c}T_{\mathrm{sf}} \\
(\mathrm{K})\end{array}$ & $\begin{array}{c}\gamma_{0} \\
\left(\mathrm{~mJ} \mathrm{~K}^{-2}\right. \\
\left.\mathrm{mol}^{-1}\right)\end{array}$ & $\begin{array}{c}\beta \\
\left(\mathrm{mJ} \mathrm{K}^{-4}\right. \\
\left.\mathrm{mol}^{-1}\right)\end{array}$ & $\begin{array}{c}T \\
(\mathrm{~K})\end{array}$ & $\begin{array}{c}\Theta_{\mathrm{D}} \\
(\mathrm{K})\end{array}$ \\
\hline $\mathrm{UFe}_{2} \mathrm{Al}_{10}$ & 28.5 & -0.3767 & 0.2747 & 11.4 & 22.5 & 0.2915 & $4.5-7.1$ & $440(10)$ \\
\hline $\mathrm{ThFe}_{2} \mathrm{Al}_{10}$ & 22.5 & -0.5171 & 0.3344 & 12.7 & 16.5 & 0.2755 & $4.9-6.9$ & $450(10)$ \\
\hline
\end{tabular}

Note that the fitting factor $\beta^{*}$ has a negative value because it contains a spin fluctuation contribution in addition to the lattice contribution and can be described as $\beta^{*}=\beta-\delta \ln T_{\mathrm{sf}}$ (where $T_{\mathrm{sf}}$ is the characteristic spin-fluctuation temperature). Hence, one can obtain $T_{\text {sf }}$ for known $\Theta_{\mathrm{D}}$. We could only roughly estimate this characteristic Debye temperature for $U$ - and Th-based aluminides, for which it amounted to $440(10)$ and $450(10) \mathrm{K}$, respectively, by an approximation of the $C_{\mathrm{p}} / T$ versus $T^{2}$ data into linear behaviour in the temperature regions also given in Table 4 and demonstrated in Fig. 6 by the dashed straight lines. Above about $5 \mathrm{~K}$, the $C_{\mathrm{p}} / T$ versus $T^{2}$ curves of both compounds behave similarly, as indicated by the differential curve of $\Delta C_{\mathrm{p}} / T$ versus $T^{2}$, which maintains an almost constant difference with increasing temperature, as is apparent in Fig. 6. This leads to an assumption that the lattice contributions for these two ternaries may be close to each other, as expected. In fact, the lattice contributions for these two ternaries are almost the same, since these two phases have the same crystal structure type and negligibly different lattice parameters, as they also have the same tetravalence and a mass difference of only $0.94 \%$. The above differential curve also implies an interesting fact: after subtraction, $\gamma(0)_{\text {extr }}\left(\mathrm{UFe}_{2} \mathrm{Al}_{10}\right)-\gamma(0)_{\text {extr }}\left(\mathrm{ThFe}_{2} \mathrm{Al}_{10}\right) \approx 3 \mathrm{~mJ} \mathrm{~K}^{-2}$ (mol. $\left.\mathrm{U}\right)^{-1}$, one obtains a very small value for this coefficient, which means that the observed enhancement of the $\gamma(0)_{\text {extr }}$ value of the Ubased aluminide is first of all caused by the iron subcell owing to the spin fluctuation mechanism. From the extrapolation to $T=0 \mathrm{~K}$ of the dashed straight lines of Fig. 6, we can estimate the difference between the values of $\gamma_{0}$ and $\gamma(0)_{\text {extr }}$ (both are also given in Table 4 ) as an enhancement due to both the electron-phonon coupling, $\lambda_{\mathrm{e}-\mathrm{ph}}$, and the spin fluctuation effect, $\lambda_{\mathrm{sf}}$, described by the equation $A \equiv$ $\gamma(0)_{\mathrm{fit}}=\gamma_{0}\left(1+\lambda_{\mathrm{e}-\mathrm{ph}}+\lambda_{\mathrm{sf}}\right)$.

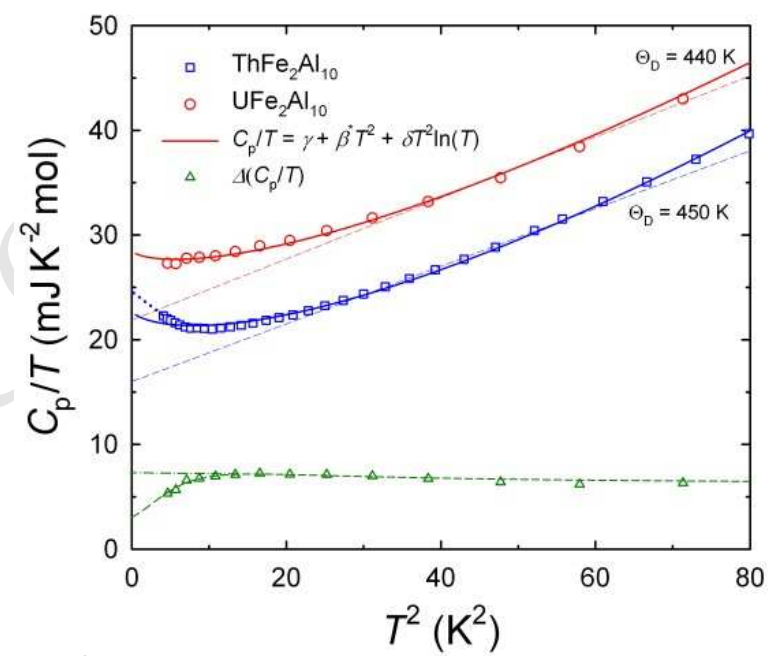

Fig. 6. The plot $C_{\mathrm{p}} / T$ versus $T^{2}$ for $\mathrm{UFe}_{2} \mathrm{Al}_{10}$ and $\mathrm{ThFe}_{2} \mathrm{Al}_{10}$. The solid line is a fitting curve to the experimental data by using Eq. (1). The dashed line presents an approximation of pure Debye contribution while the dotdashed line is the difference $\Delta\left[C_{\mathrm{p}}(T)_{\mathrm{U}}-C_{\mathrm{p}}(T)_{\mathrm{Th}}\right] T^{-1}$.

For both these aluminides, the sum $\left(\lambda_{\mathrm{e}-\mathrm{ph}+} \lambda_{\mathrm{sf}}\right)$ is equal to about 0.32 . Clearly, this value of the coupling was not enough to cause superconductivity to arise in these compounds down to $2 \mathrm{~K}$. Only the application of a sufficiently strong magnetic field can quench spin fluctuations and just reduce $\lambda_{\text {sf. }}$ It is 
also remarkable that the $\gamma(0)_{\text {extr }}$ values for both aluminides containing $\mathrm{Y}$ or Th are enhanced by magnetic fluctuations and have similar values, that is 29.0 [32] and $25.7 \mathrm{~mJ} \mathrm{~K}^{-2} \mathrm{~mol}^{-1}$, respectively.

As mentioned above, in order to analyse the $C_{\mathrm{p}}(T)$ function of the nonmagnetic reference, $\mathrm{ThFe}_{2} \mathrm{Al}_{10}$, shown in Fig. 5, one should estimate the temperature variations of three components: the Debye $C_{\mathrm{D}}(T)$, Einstein $C_{\mathrm{E}}(T)$, and electronic $C_{e}(T)$ one. For this purpose, we have first analysed the $C_{\mathrm{p}}\left(\mathrm{ThFe}_{2} \mathrm{Al}_{10}\right) / T^{3}$ versus $\ln T$ dependence, which was finally deconvoluted and delineated, as displayed in Fig. 7(a).
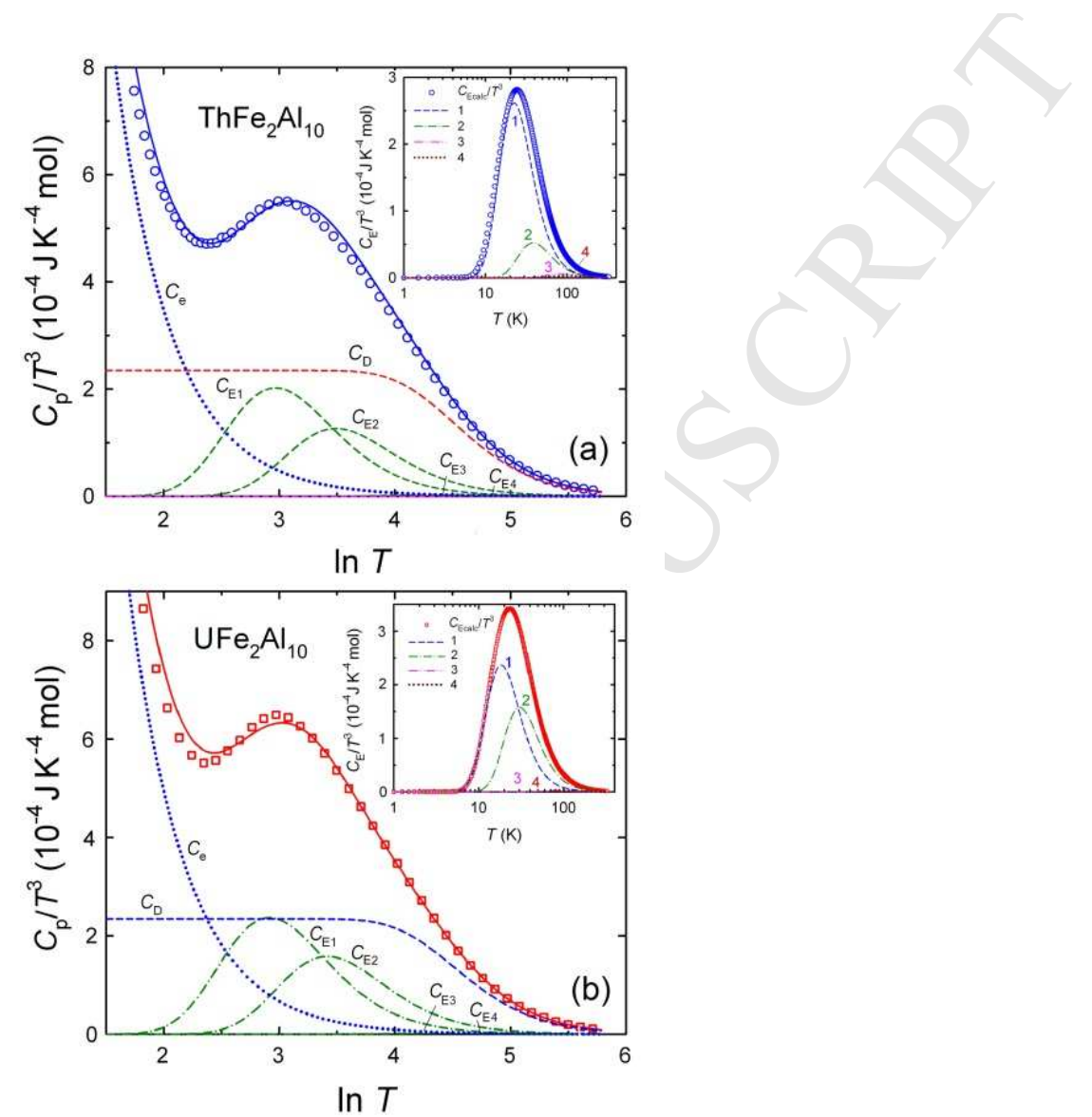

Fig. 7. The dependencies $C_{\mathrm{p}}(T) T^{-3}$ versus $\ln T$ for (a) $\mathrm{ThFe}_{2} \mathrm{Al}_{10}$ together with deconvoluting this function into contributions: $C_{\mathrm{D}}, C_{\mathrm{Ei}}\left(\mathrm{i}=1,2,3 \mathrm{i}\right.$ 4) and $C_{\mathrm{e}}$ constituents and (b) $\mathrm{UFe}_{2} \mathrm{Al}_{10}$. In the insets to (a) and (b), we present the fitting of four $C_{\mathrm{Ei}}$ Vs. $\log T$ extracted curves.

As shown, this function goes through a broad maximum at $T_{\max }$, indicating that except for the acoustic modes, we have to do certainly with the optical ones. The fitting has made it possible to attribute $\Theta_{\mathrm{D}}(\approx 450 \mathrm{~K})$ and four Einstein temperatures, two $\left[\Theta_{\mathrm{E} 1}(=96 \mathrm{~K})\right.$ and $\left.\Theta_{\mathrm{E} 2}(=162 \mathrm{~K})\right]$ of larger intensities and two $\left[\Theta_{\mathrm{E} 3}(=398 \mathrm{~K})\right.$ and $\left.\Theta_{\mathrm{E} 4}(=629 \mathrm{~K})\right]$ of small intensities. The goodness of the Einstein model fit can be clearly seen in the separate curve presented as the inset to Fig. 7(a). The final fitting was that of the lowest temperature tail to the experimental data. The assumed value $\gamma(0)=22.5$ $\mathrm{mJ} \mathrm{K}^{-2} \mathrm{~mol}^{-1}$ results in a track of $C_{\mathrm{e}}(T)$ slightly higher than the experimental points. Taking the extrapolated value of $\gamma(0)_{\text {extr }}\left(25.0 \mathrm{~mJ} \mathrm{~K}^{-2} \mathrm{~mol}^{-1}\right)$ into account made the fitting considerably worse. In turn, in Fig. 7(b) we display the aforementioned dependence for $\mathrm{UFe}_{2} \mathrm{Al}_{10}$ with extracted parameters similar to those for $\mathrm{ThFe}_{2} \mathrm{Al}_{10}$. They are as follows: $\Theta_{\mathrm{D}}(\approx 440 \mathrm{~K}), \Theta_{\mathrm{E} 1}(=91 \mathrm{~K}), \Theta_{\mathrm{E} 2}(=150 \mathrm{~K}), \Theta_{\mathrm{E} 3}(=$ $421 \mathrm{~K})$ and $\Theta_{\mathrm{E} 4}(=432 \mathrm{~K})$. Also here the $\gamma(0)_{\mathrm{extr}} \approx \gamma(0)_{\text {fit }}$ value again gave a higher track than the experimental points. Some differences are seen between Th- and U-aluminides in both the electronic heat contribution and the intensities of the main Einstein modes. Moreover, below we will also discuss the possibility that some low-frequency Einstein modes may also exist which at present remain practically undetected within the framework of an applied simple analysis of the specific heat data, as 
has been done in this paper, and also due to the lack of information from more advanced measurements using continuous curves and not sampling point by point.

\subsubsection{Magnetic susceptibility and magnetization}

The results of magnetic examination of $\mathrm{ThFe}_{2} \mathrm{Al}_{10}$ single crystals are shown in Fig. 8. In this figure, we display the temperature dependencies of the susceptibility, $\chi_{\| a, c}(T)$, measured along both crystallographic directions, $a$ and $c$. As can be seen, above about $50 \mathrm{~K}$, the corresponding curves show only weakly temperature-dependent Pauli-like paramagnetism with small anisotropy. However, at about $250 \mathrm{~K}$ some inflection point is marked for these two curves instead of the expected smooth weak-temperature dependence.

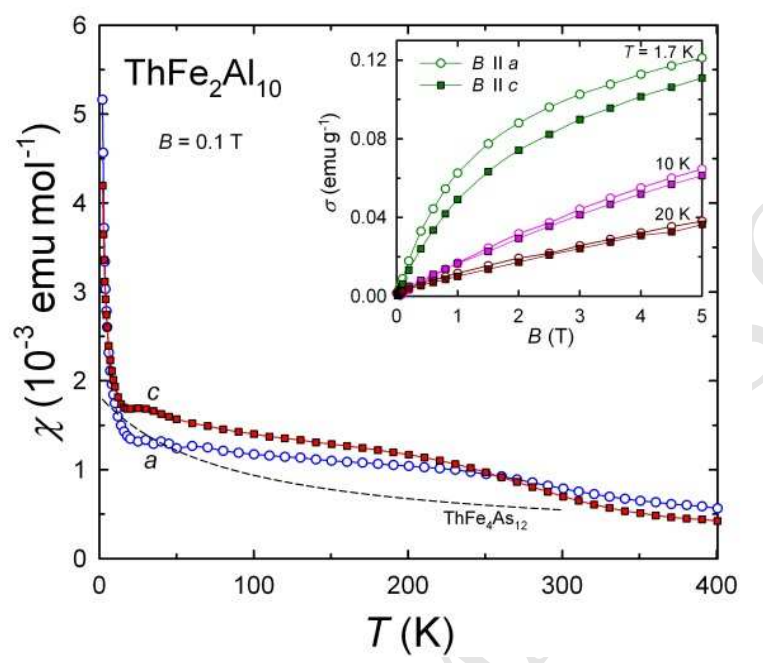

Fig. 8. Temperature dependencies of molar magnetic susceptibility of $\mathrm{ThFe}_{2} \mathrm{Al}_{10}$ measured along the $a$ and $c$ axes, compared to that of $\mathrm{ThFe}_{4} \mathrm{As}_{12}$ taken from Ref. 35. In the inset, there is shown the low-temperature magnetization $\sigma$ (in emu $\mathrm{g}^{-1}$ units) versus applied fields measured at several selected temperatures also along the $a$ and $c$ axes.

On the other hand, below about $50 \mathrm{~K}$, a very small susceptibility anomaly is also seen along the measured two directions, which have their local maxima at about $30 \mathrm{~K}$. When the temperature is decreased further, both maxima are followed by a rapid rise of the susceptibility. For any Pauli-like paramagnet such a rapid increase in the susceptibility values at low temperatures is usually interpreted as being caused by very small contamination in the studied sample by indefinable Curie-Weiss paramagnet. However, in such a case, the magnetization should be linear in magnetic field, which is not observed, as the inset to Fig. 8 indicates. Instead, the magnetization of $\mathrm{ThFe}_{2} \mathrm{Al}_{10}$ at $1.7 \mathrm{~K}$ is strongly curvilinear, reaching values of 0.013 and $0.012 \mu_{\mathrm{B}}$ /f.u. for $\boldsymbol{B} \| a$ or $\boldsymbol{B} \| c$, respectively, in an applied field of $5 \mathrm{~T}$. This behaviour suggests that the Fe atoms exhibit some tendency to magnetic ordering at very low temperature, but the magnetic moment per Fe-atom is quite small, in a similar way to that of the $\mathrm{Co}$ atoms in $\mathrm{LaCoGe}_{3}$ [33] or $\mathrm{LaCo}_{0.96} \mathrm{Ge}_{2}$ [34]. The low-temperature strong curvilinear variation of the magnetization of $\mathrm{ThFe}_{2} \mathrm{Al}_{10}$ and a small magnetic moment indicate that the electron transfer from the Th atom to Fe nearly fills up the $3 d$ bands, giving rise, however, to the small magnitude of the magnetic moment at the Fe sites and the occurrence of a spin-fluctuation effect, according to the specific heat data described above. At higher temperatures, the magnetization is already linear in field. For comparison, in Fig. 8 we have also plotted the magnetic data of the skutterudite $\mathrm{ThFe}_{4} \mathrm{As}_{12}$ [35], for which the temperature variation of the susceptibility, $\chi(T)$, resembles that of $\mathrm{ThFe}_{2} \mathrm{Al}_{10}$, although the atomic Fe:Th ratio is twice as large for the arsenide. In such a situation, one would expect an insufficient number of electrons from the Th shell to fill up the $3 d$ shell of iron completely. Nevertheless, the magnetization of that skutterudite at $2 \mathrm{~K}$ is almost linear in field (see the inset of Fig. 2 in Ref. [35]), which indicates the lack of magnetic order, and the specific heat divided by temperature yields a straight line against $T^{2}$ down to $2 \mathrm{~K}$. On the other hand, the lowtemperature magnetization curves presented in the inset of Fig. 8 in this paper for $\mathrm{ThFe}_{2} \mathrm{Al}_{10}$ are reminiscent of those for $\mathrm{YFe}_{2} \mathrm{Al}_{10}$ (see the inset 1 in Ref. [36]), which was found to be very close to a quantum critical point (QCP). In such a situation, the critical fluctuations associated with this $T=0 \mathrm{~K}$ 
transition should just lead to power-law temperature divergences of the susceptibility and specific heat divided by $T$ as a function of $\ln T$ at the lowest temperature, which was really observed in this Y-based aluminide [36]. As already pointed out above, the $C_{\mathrm{p}} / T$ values of $\mathrm{ThFe}_{2} \mathrm{Al}_{10}$ also start to increase at the lowest temperatures in the $\ln T$ manner, confirming the closeness of this compound to the QCP. As shown above, the characteristic temperature $T_{\text {sf }}$ is rather low and amounts to roughly $11 \mathrm{~K}$.

\subsubsection{Transport properties}

Electrical resistivity and transversal magnetoresitivity (TMR), defined as $\Delta \rho / \rho(0)=[\rho(B, T)-$ $\rho(0, T)] / \rho(0, T)$, were based on AC measurements using bar-shaped samples cut from a single crystal of an irregular form. In Fig. 9 we have first presented the $\rho(T)$ functions for the current $\boldsymbol{J}$ directed along the $a$ or $c$ direction. As can be seen from this figure, both of these temperature dependencies exhibit a minimum at about $25 \mathrm{~K}$ and form an upturn below that temperature. The occurrence of the lowtemperature minimum in $\rho(T)$ may point to the presence of the two-channel Kondo effect derived from structural two-level energy systems [37], as discussed in detail for ThAsSe [38], for example. The obtained residual resistivity ratio (RRR) is rather small (about 4.5), but it is usually difficult for any thorium compound to obtain a high-quality single crystal. The above results point to a typical metallic behaviour of $\mathrm{ThFe}_{2} \mathrm{Al}_{10}$ with a positive curvature at high temperatures. We have carried out a fitting of the experimental data to the equation:

$$
\rho(T)=\rho_{0}+\rho_{\mathrm{BG}}(T)+K T^{3}+C \ln T
$$

where $K T^{3}$ is Mott's $s-d$ scattering term and $\rho_{\mathrm{BG}}(T)$ with the coefficient $n=3$ is the well-known BlochGrüneisen formula:

$$
\rho_{\mathrm{BG}}(T)=4 \mathrm{R} \Theta_{\mathrm{D}}^{\mathrm{R}}\left(T / \Theta_{\mathrm{D}}^{\mathrm{R}}\right)^{n} \int_{0}^{\Theta_{\mathrm{D}}^{\mathrm{R}} / T} \frac{z^{n} \mathrm{dz}}{\left(e^{z}-1\right)\left(1-e^{-z}\right)}
$$

where $\mathrm{R}$ is the coefficient of the phonon term and $C$ is a constant value. As Fig. 9 indicates, we found a very good fit with $\Theta_{\mathrm{D}}^{\mathrm{R}}=261$ and $340 \mathrm{~K}$ for $J \| a$ and $J \| c$, respectively, and by adding the logarithmic term due to a Kondo-like contribution. We treat Eq. (2) as an analytical one and we will use it in our further analysis of the magnetoresistivity. The constant parameters are given in Table 5.

Table. 5 The Bloch-Grüneisen parameters and Mott's and Kondo constants.

\begin{tabular}{cccccc}
\hline $\mathrm{ThFe}_{2} \mathrm{Al}_{10}$ & $\begin{array}{c}\rho_{0} \\
(\mu \Omega \mathrm{cm})\end{array}$ & $\begin{array}{c}\mathrm{R} \\
\left(\mu \Omega \mathrm{cm} \mathrm{K}^{-1}\right)\end{array}$ & $\begin{array}{c}\Theta_{\mathrm{D}}^{\mathrm{R}} \\
(\mathrm{K})\end{array}$ & $\begin{array}{c}K \\
\left(\mu \Omega \mathrm{cm} \mathrm{K}^{-3}\right)\end{array}$ & $\begin{array}{c}C \\
(\mu \Omega \mathrm{cm})\end{array}$ \\
\hline $\boldsymbol{J} \| a$ & 69.5 & 0.2474 & 261 & $-9.246 \times 10^{-7}$ & -3.60 \\
$\boldsymbol{J} \| c$ & 14.4 & 0.1129 & 340 & $-7.677 \times 10^{-8}$ & -5.10 \\
\hline
\end{tabular}

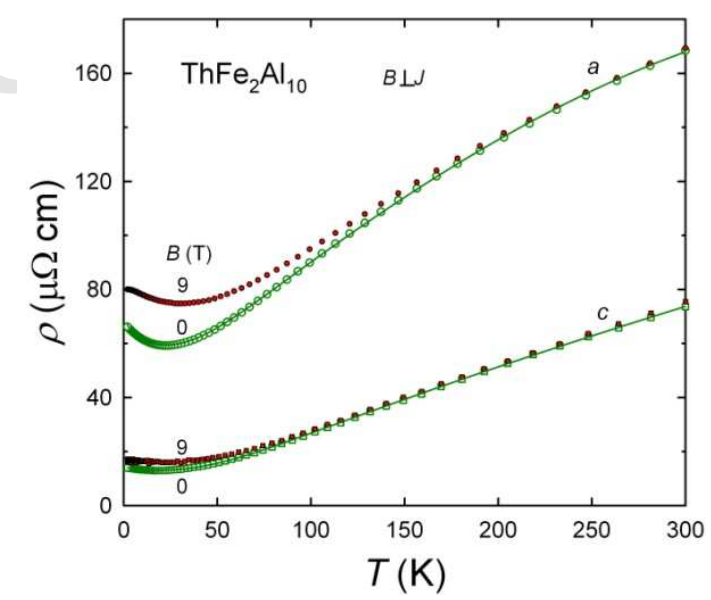

Fig. 9. Temperature dependencies of the resistivity in $\mathrm{ThFe}_{2} \mathrm{Al}_{10}$ measured along the $a$ and $c$ axes in zero field and 9 T. Solid lines denote fitting to Eq. (2). 
As Fig. 9 indicates, the zero-field $\rho(T)$ curves found for the $\boldsymbol{J} \| a$ and $J \| c$ cases and the corresponding TMR curves taken with an applied magnetic field of $9 \mathrm{~T}$ cover each other at higher temperatures. However, at low temperatures, TMR has an apparent positive effect, which is especially well marked in the case of $\boldsymbol{J} \| a$. In order to illustrate this effect more distinctly, in Fig. 10 we have plotted the low-temperature behaviour of the electrical resistivity (left-hand curves) as well as the $9 \mathrm{~T}$ magnetoresistivity results in the form of $\Delta \rho / \rho(0)(\%)$ (right-hand curves), taken for both directions of the current $\boldsymbol{J}$ and using an enhanced scale. Considering the low-temperature variation of the resistivity for the $J \| a$ case, measured at zero field and $9 \mathrm{~T}$, as well as the corresponding TMR, one can see the high accuracy of all these measurements, where the size of the experimental points (marked by circles) determines the maximum magnitude of the experimental error.

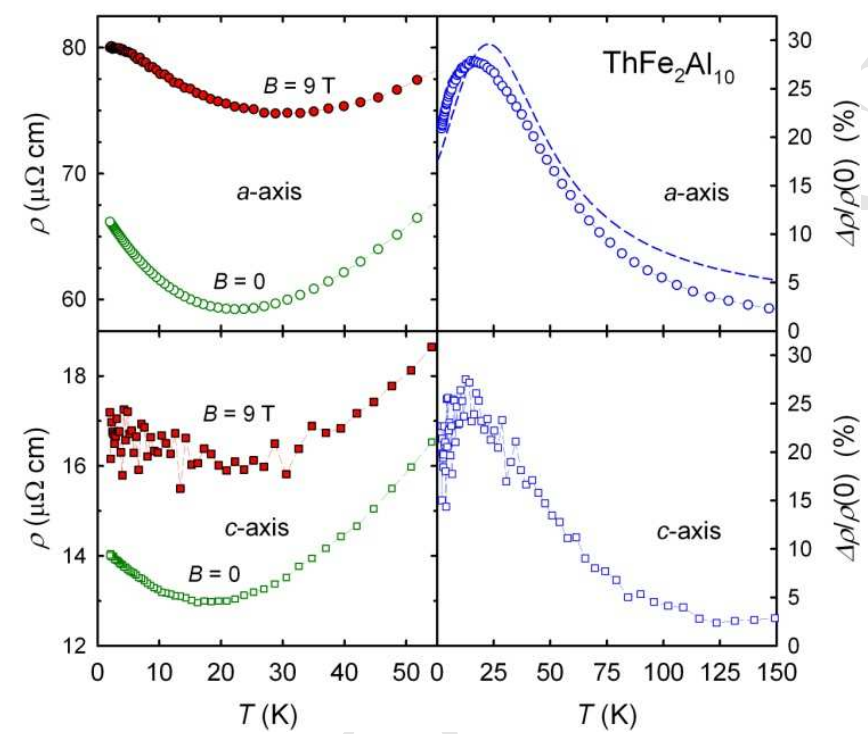

Fig. 10. The low-temperature resistivity taken in zero field and $9 \mathrm{~T}$ on an enlarged scale (left-hand panels) and reduced transverse magnetoresistivity (at $9 \mathrm{~T}$ ) vs. temperature (right-hand panels) of $\mathrm{ThFe}_{2} \mathrm{Al}_{10}$ measured along the $a$ and $c$ axes. The dashed line presents an exemplary temperature dependence of TMR calculated using Eq. (4).

Quite a different situation takes place when the measurements are performed along the $c$-axis in an applied magnetic field. An inspection of the bottom of this figure reveals a quite interesting behaviour of the resistivity measured along that axis at a field of $9 \mathrm{~T}$ and hence also the temperature dependence of the magnetoresistivity. Although the accuracy of electrical resistivity measurements at zero-field measurements is the same as that along the $a$-axis, the scattering of the experimental points becomes far beyond the possible committed errors when one applies the magnetic field of $9 \mathrm{~T}$. Such an effect signals that under an applied magnetic field the Th atom, imprisoned in the oversized $\mathrm{Al}-\mathrm{Fe}$ cage and exhibiting metallic character, experiences some variation in its crystallographic position just along the $c$-axis, that is, the direction of the zigzag chains formed by the Th atoms inside such a highly anisotropic framework (see e.g. Fig. 3(b) in Ref. [39]). In consequence, a large scattering in the resistivity data is observed at low temperatures. This effect, however, decreases when the temperature is raised above about $30 \mathrm{~K}$ and then the $9 \mathrm{~T}$ resistivity becomes smooth like the zero-field data. Interestingly, such behaviour was not observed in the case of isostructural $\mathrm{UFe}_{2} \mathrm{Al}_{10}$, where, however, the uranium atom has an ionic character and the chemical bonding with the cage atoms is considerably stronger than that in Th-based aluminide [15]. The phenomenon of strong scattering of the resistivity data at low temperatures under a magnetic field was reported for the first time for the dodecaboride $\mathrm{UB}_{12}$ [40], which is also a cage compound, where the metallic uranium central atom is surrounded by an isotropic framework of 24 boron atoms. This finding sheds more light onto the cubic crystal structure of the dodecaborides, where the Einstein oscillators are sufficiently free around their sites due to a very weak chemical bonding of the uranium atom with its surroundings. As a result, atomic rattling takes place inside the isotropic B-framework, while in the case of the orthorhombic structure 
of $\mathrm{ThFe}_{2} \mathrm{Al}_{10}$ such rattling is associated, so far, with only one of the crystallographic directions, namely along the $c$-axis, although we do not know the electrical resistivity data along the $b$-axis, which remain to be measured. This is because the shape of our single crystals did not allow us to perform measurements along this direction.

Previously, the phenomenon of low-temperature rattling vibration was also observed in specific heat measurements, for example in the case of the cage compound $\mathrm{Ga}_{\mathrm{x}} \mathrm{V}_{2} \mathrm{Al}_{20}$ [41]. This feature was reported to be associated with a local mode approximated by an Einstein low-frequency mode with the Einstein temperature $\Theta_{\mathrm{E}} \approx 20 \mathrm{~K}$. Thus, such a low energy mode is favourable for coupling strongly with conduction electrons due to the electron-rattler $(e-r)$ interaction, which creates anharmonicity as well as a concave low-temperature dependence of the resistivity, also described by the authors of Ref. [41] using almost the same value of $\Theta_{\mathrm{E}}$. Based on this fact, one can conclude that as a result of the above $e-r$ interaction the magnetoresistivity is also influenced by the rattling vibration, giving rise to the observed scattering of the experimental points. Unfortunately, we were not able to determine such a low value of $\Theta_{\mathrm{E}}$ for $\mathrm{ThFe}_{2} \mathrm{Al}_{10}$ from our physical measurements because of other low-temperature components that also exist in this compound, such as spin-fluctuation and Kondo-like ones. Furthermore, the temperature dependence of the relative magnetoresistivity of this Th-based aluminide, shown in the right-hand parts of Fig. 10, goes through a maximum at about $20 \mathrm{~K}$ for both crystallographic directions $a$ and $c$. Interestingly, the TMR measured along three directions, $a$, $b$, and $c$, for the uranium-based aluminide shows such a maximum merely for $J \| c$ (see Fig. 11 in Ref. [15]). At the same time, on the smoothed $9 \mathrm{~T}$ curve measured along the $a$-axis, displayed in Fig. 10, we marked the results of those data that best fitted Eq. (4) (dashed line) [42]:

$$
\frac{\Delta \rho}{\rho(0)}=\frac{B^{2}}{\mathrm{a}[\rho(0, T)]+\mathrm{b} B^{2}}
$$

where $\mathrm{a}$ and $\mathrm{b}$ are the field- and temperature-independent parameters depending exclusively on conduction electron properties. $\rho(0, T)$ is the total resistivity at zero field, which is given analytically by Eq. (2). The parameters a and $\mathrm{b}$ (for the $a$ axis) are $0.230 \mathrm{~T}^{2}(\mu \Omega \mathrm{cm})^{-1}$ and -0.135 , respectively. Thus, the dashed line in Fig. 10 is the result of least-squares fitting of Eq. (4) to the magnetoresistivity data. The above formula describes the so-called normal magnetoresistivity, that is, the influence of the magnetic field on the conduction electron trajectories (Lorentz effect). This mechanism always gives rise to positive TMR and increases the magnetoresistivity while lowering the temperature at a fixed finite field. This effect is due to the decrease in electron-phonon scattering as the temperature is lowered [42].

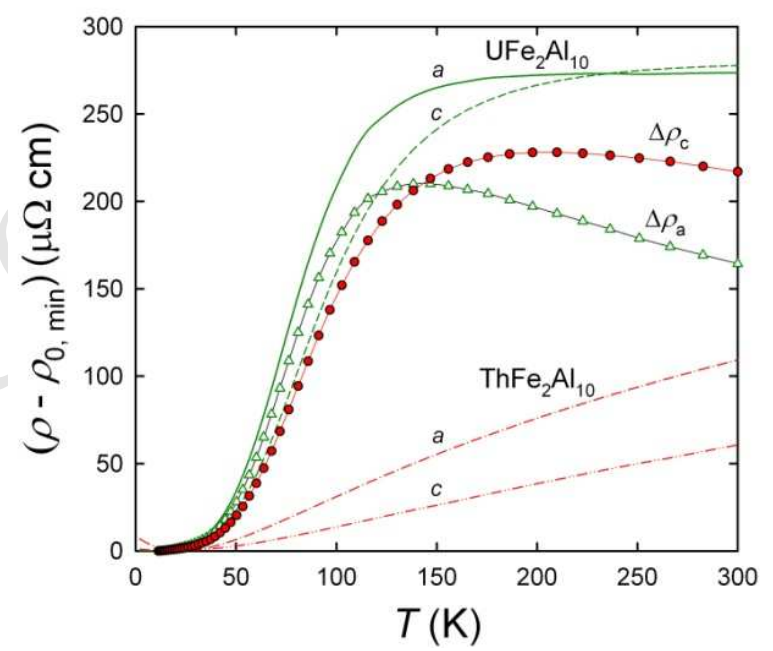

Fig. 11. Temperature dependencies of the resistivity measured along the $a$ and $c$ axes for $\mathrm{UFe}_{2} \mathrm{Al}_{10}($ data taken from Ref. 15) and $\mathrm{ThFe}_{2} \mathrm{Al}_{10}$ (this paper) after subtracting the residual resistivities $\rho_{0}$ and $\rho_{\text {min }}$, respectively. The curves following the open triangles ( $a$-axis) and closed circles ( $c$-axis) are the temperature variations of differences in resistivities $\Delta \rho_{\mathrm{i}}(\mathrm{i}=a$ - and $c$-directions $)$ between the corresponding curves presented in this figure. 
The obtained temperature dependencies of $\rho(T)_{\mathrm{Th}}$ for the directions $\boldsymbol{J} \| a$ and $J \| c$ of $\mathrm{ThFe}_{2} \mathrm{Al}_{10}$ were then used to carry out an analysis of such dependencies of $\rho(T)_{\mathrm{U}}$ for $\mathrm{UFe}_{2} \mathrm{Al}_{10}$ [15] measured along the same directions of $\boldsymbol{J}$. By subtracting $\rho_{\min }$ from $\rho(T)_{\mathrm{Th}}$ and $\rho_{0}$ from $\rho(T)_{\mathrm{U}}$ and treating $\left[\rho(T)_{\mathrm{Th}}-\right.$ $\left.\rho_{\min }\right]$ as the phonon contribution, we were able to determine the temperature dependencies of the differences $\Delta \rho_{a}(T)$ and $\Delta \rho_{c}(T)$, which are the magnetic contributions to the electrical transport of $\mathrm{UFe}_{2} \mathrm{Al}_{10}$. All of these functions are presented in Fig. 11.

In the next step of our analysis, we undertook a task to estimate all the possible components in the extracted magnetic part of the electrical resistivity of $\mathrm{UFe}_{2} \mathrm{Al}_{10}$ by considering various scattering processes, which are partially competing and may therefore result in the observed magnetic part of the temperature dependences of resistivity taken as an average of two crystal directions, as shown in Fig. 12. In the following we ignore the anisotropy and focus instead on the main features of the experimental curve averaged over the $a$ and $c$ directions. For the compound under study whose magnetic and thermal properties imply the nearly localized picture of the $5 f$ electrons [15], the $s-f$ interaction [43, 44], as appearing in the lowest-order Born approximation, is the first candidate to elucidate the increase in resistivity with temperature up to the broad maximum at $150-200 \mathrm{~K}$. It is expressed as:

where

$$
\rho_{s-f}=\rho_{s-f 0} \operatorname{Tr}(P Q)
$$

$$
\begin{gathered}
Q_{\alpha \beta}=1 / 2 \mid\left\langle\alpha\left|J^{+}\right| \beta>\left.\right|^{2}+1 / 2|\langle\alpha|J| \beta\rangle|^{2}+\left.\left\langle\alpha\left|J^{2}\right| \beta\right\rangle\right|^{2},\right. \\
P_{\alpha \beta}=\frac{1}{Z} \frac{2}{e^{E_{\alpha} / T}+e^{E_{\beta} / T}} .
\end{gathered}
$$

In the above formulae, $\alpha$ and $\beta$ are the quantum numbers identifying components of the crystal field states; $E_{\alpha}$ are the corresponding energies in $\mathrm{K}$; and $J^{+}, J^{-}$, and $J^{z}$ are the momentum operator components. $\rho_{s-f 0}$ is a factor proportional to the square of the $s-f$ interaction constant and inversely proportional to the Fermi energy.

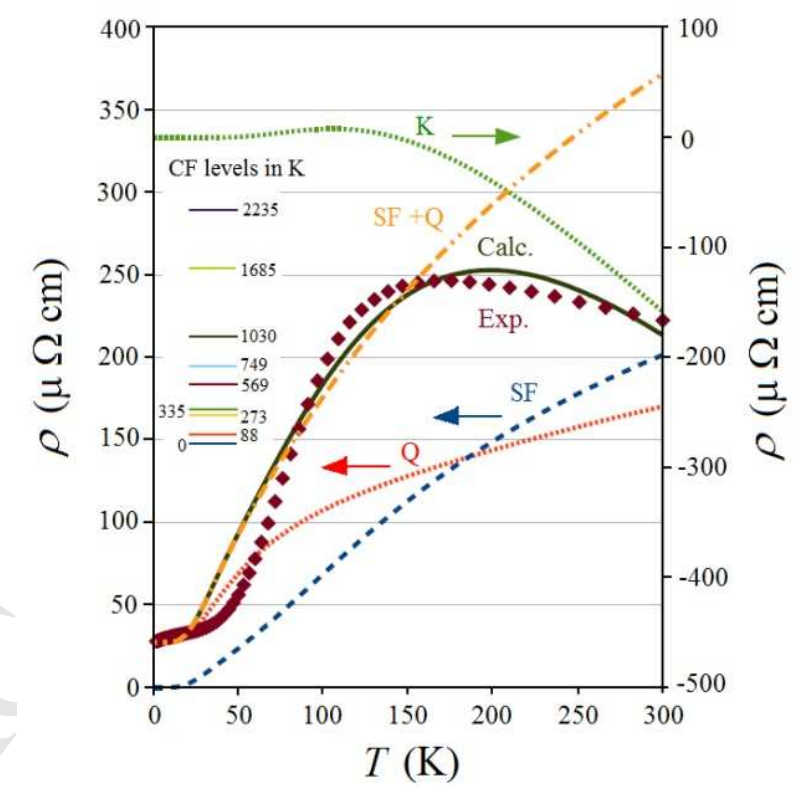

Fig. 12. $\mathrm{UFe}_{2} \mathrm{Al}_{10}$ : The experimental curve averaged over two crystal directions (diamonds) versus the theoretical temperature dependence of the magnetic contribution to the resistivity (line) and its components: (SF) - the $s$ - $f$ scattering, (Q) - the Coulomb quadrupole scattering and $(\mathrm{K})$ - the correction due to the higher order scattering processes. The CF levels scheme from Ref. 15 is shown as well.

Indeed, with the CF states obtained previously [15], the $s-f$ scattering provides a contribution that increases in the whole temperature range, indicated by the line SF in Fig. 12. Nevertheless, it cannot account for either the measured non-zero residual resistivity $\rho_{0}=\rho(T \rightarrow 0)$ or the mentioned decrease above the maximum at $\sim 170 \mathrm{~K}$, and therefore other mechanisms must be taken into account. Apart from lattice imperfections, the lowest-order mechanism that gives a non-zero residual contribution to 
the $\rho_{0}$ is the scattering of the conduction electrons by electric quadrupoles formed by the $5 f$ shell electrons, which generates the following component $[45,46]$ :

where

$$
\rho_{Q}=\rho_{Q 0} \operatorname{Tr}(P O)
$$

$$
O_{\alpha \beta}=\sum_{q=-2, \ldots, 2}\left|\left\langle\alpha\left|C_{q}^{(2)}\right| \beta\right\rangle\right|^{2},
$$

$C_{q}^{(2)}$ is the second rank normalized spherical harmonic operator, the matrix $P_{\alpha \beta}$ is given by Eq. (7), and $\rho_{Q 0}$ is a factor proportional to the electron-quadrupole interaction constant and is dependent on other material constants. The corresponding line (Q) in Fig. 12 represents the upper limit for this particular contribution, since the whole of the observed $\rho_{0} \sim 27 \mu \Omega \mathrm{cm}$ has been attributed to $\rho_{Q 0}$, disregarding the influence of the lattice imperfections and impurities.

As seen in Fig. 12, both mechanisms, $s-f$ and Q, produce monotonic contribution in the whole temperature range (and far above it). This is a consequence of the crystal field splitting being much greater than the thermal energy. There exist levels whose thermal population has the capacity to grow for any temperature in the range under consideration. These two contributions together, $\rho_{s-f}+\rho_{Q}$, reproduce the experimental points well up to $\sim 90 \mathrm{~K}$ only. The singlet ground state separated by $88 \mathrm{~K}$ from the first excited level prevents other scattering mechanisms from being efficient in this range. Note that the coefficients $\rho_{Q 0}$ and $\rho_{s-f 0}$ are the only free parameters of the contributions $s-f$ and $\mathrm{Q}$ since the shape of the corresponding lines is completely determined by the crystal field levels. In practice, this means that the $\rho_{s-f 0}$ has to be adjusted to the experimental points only because $\rho_{Q 0}$, or rather its upper limit, as explained above, is determined by the residual experimental resistivity $\rho_{0}$.

Taking into account the whole temperature range, it is clear that the slight decrease of the resistivity above the maximum at $170 \mathrm{~K}$ still requires an explanation at the moment. The problem is that many higher-order terms in the scattering potential expansion are possible. Their identification in such a complex system and estimation would be very difficult. Therefore, guided by the shape of the experimental curve above $100 \mathrm{~K}$, we assume that their overall contribution in the form of the logarithmic decrease is the weakest one, with the magnitude and slope controlled by the parameters $\rho_{\mathrm{K} 0}$ and $D$ :

$$
\rho_{\mathrm{K}}=\rho_{\mathrm{K} 0} e^{-E / T} \ln (T / D) .
$$

In addition, the singlet ground state implies inclusion of the Boltzmann-like temperature-dependent weight that switches on the component above a certain temperature, adjusted by the parameter $E$. The parameter $D$ can be understood as a kind of measure of the freezing of the degrees of freedom due to a reconstruction of the many-electron states by the scattered conduction electron in the higher-order processes. Thus, the experimental averaged magnetic (electronic) resistivity has been reproduced by simultaneous fitting of the weight $\rho_{\mathrm{K} 0}$ as well as the parameters $E$ and $D$ of the total theoretical resistivity:

$$
\rho=\rho_{s-f}+\rho_{Q}+\rho_{\mathrm{K}}
$$

with $\rho_{s-f}$ and $\rho_{Q}$ being determined as explained above. In such a formulation, it is clear that the component K (10) plays a dual role. It not only captures possible higher-order scattering processes but also compensates the $\rho_{s-f}$ and $\rho_{Q}$ contributions, which lose their original sense at elevated temperatures (at which the more involved processes take place). This explains the high value of $\rho_{\mathrm{K} 0}=-675 \mu \Omega \mathrm{cm}$. The remaining parameters are the following: $E=343 \mathrm{~K}, D=144 \mathrm{~K}, \rho_{s-f 0}=46 \mu \Omega \mathrm{cm}$, and $\rho_{Q 0}=4509$ $\mu \Omega \mathrm{cm}$. The last parameter may be overestimated because we attributed the whole residual resistivity to the quadrupole scattering. Nevertheless, a strong scattering of the conduction electrons by the quadrupole component of the $5 f$ electronic charge, comparable with the $s-f$ contribution, has been observed for compounds such as $\mathrm{PrOs}_{4} \mathrm{Sb}_{12}$ [46], among others. Thus, the interaction constant included in $\rho_{Q 0}$ must be large enough to compensate small values of the $O$ matrix elements (9) in comparison with $Q$ ones (6). We hope that a similar analysis (which is in progress) for other members of the series, $\mathrm{URu}_{2} \mathrm{Al}_{10}$ and $\mathrm{UOs}_{2} \mathrm{Al}_{10}$, will shed new light on this problem. It will be reported soon [47].

\subsubsection{Seebeck coefficient}

The temperature dependencies of thermoelectric power (TEP or $S$ ) of $\mathrm{ThFe}_{2} \mathrm{Al}_{10}$ were also found along the $a$ and $c$ axes. As Fig. 13 illustrates, TEP is anisotropic for these directions and negative within the 
whole temperature range measured. Negative values of TEP indicate that the dominant carriers here are electrons. Interestingly, an apparent linear region in temperature behaviour starts above about $30 \mathrm{~K}$ and finally reaches the values of -25 and $-16 \mu \mathrm{V} \mathrm{K}^{-1}$, respectively, at RT. However, the extrapolations of the corresponding straight lines to $T=0 \mathrm{~K}$ do not yield the zero-value of TEP but intersect the y axis at $S_{a}(0)=-1.55 \mu \mathrm{V} \mathrm{K}^{-1}$ and $S_{c}(0)=-2.35 \mu \mathrm{V} \mathrm{K} \mathrm{K}^{-1}$. Therefore, the above straight lines at low temperatures change into curves and achieve, as predicted by theory, their zero value at $T=0 \mathrm{~K}$. This feature is shown in the inset of Fig. 13 on an enhanced scale below $8 \mathrm{~K}$. The values of $S T^{-1}$ (for $T \rightarrow$ $0)$ tangents in the $a$ and $c$ directions are -0.143 and $-0.351 \mu \mathrm{V} \mathrm{K} \mathrm{K}^{-2}$, respectively. In turn, the corresponding slopes in the high-temperature range are -0.0783 and $-0.0450 \mu \mathrm{V} \mathrm{K}^{-2}$, respectively. This type of behaviour is typical of simple metals, for which TEP can be written as a sum:

$$
S(T)=S_{\mathrm{d}}(T)+S_{\mathrm{g}}(T)
$$

where $S_{\mathrm{d}}$ and $S_{\mathrm{g}}$ are thermoelectric powers due to electron diffusion and phonon drag, respectively. The former component is given by the standard one-band Mott's formula [47]:

$$
S_{\mathrm{d}}(T)=\xi\left(k_{\mathrm{B}}^{2} \pi^{2} T\right) /\left(3 e E_{\mathrm{F}}\right)
$$

where $k_{\mathrm{B}}$ is the Boltzmann constant and $e$ is an electron charge. The $\xi$ parameter depends on the mechanism of the electron scattering and its value is usually taken to be between 2 and 3 [48]. Assuming, however, different values of $\xi$ in our case, for various directions of measurements, one would be able to obtain a proper isotropic value of $E_{\mathrm{F}}$ using Eq. (13) by probing different slopes of $S_{a}(T)$ and $S_{\mathrm{c}}(T)$. Furthermore, the observed deviation of the total TEP from linearity at low temperatures is usually ascribed to the phonon-drag term $S_{\mathrm{g}}$. On the other hand, the $S_{\mathrm{g}}$ is also diminished at low temperatures by strong scattering between phonons and impurities. This fact implies that only examination of very pure crystals allows one to observe a pronounced phonon-drag maximum.

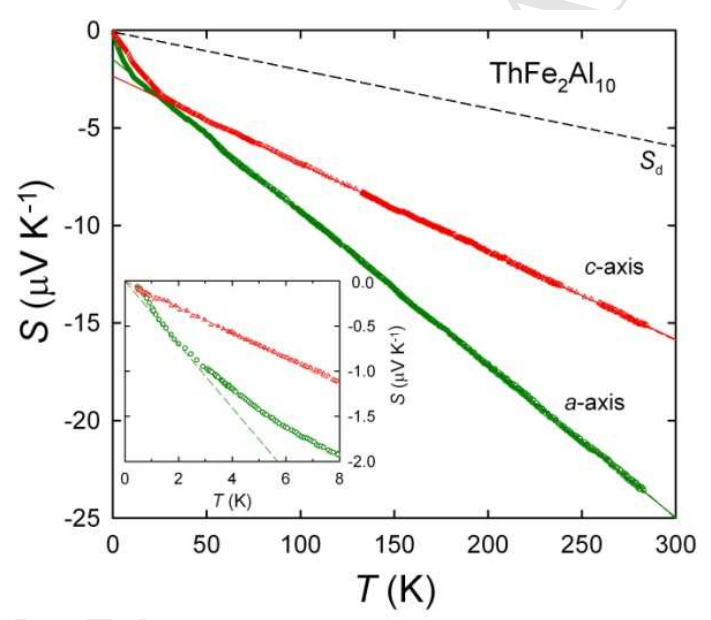

Fig. 13. Temperature variations of thermoelectric power of $\mathrm{ThFe}_{2} \mathrm{Al}_{10}$ measured along the $a$ and $c$ axes. The black dashed line denotes $S_{\mathrm{d}}(T)$ estimated from Eq. (14). The inset shows the low-temperature deviations from the high-temperature straight lines of the $S(T)$ behaviour.

Quite a different situation takes place in the isostructural $\mathrm{UFe}_{2} \mathrm{Al}_{10}$, where this system contains a localized magnetic moment, but without a sign of forming a magnetic ordering state down to the lowest temperature [15]. The TEP behaviour of this ternary compound is very anisotropic and exhibits broad corresponding maxima at about $70 \mathrm{~K}$ for the $a, b$, and $c$ axes. In this case, there are also two main contributions: one already described above, that is, a diffusion term, $S_{\mathrm{d}}(T)$, and the other one is the crystal-field term, $S_{\mathrm{CF}}(T)$.

To estimate the overall magnitude of the temperature dependence of TEP in $\operatorname{ThFe}_{2} \mathrm{Al}_{10}$, we have used the Mott model for diffusion TEP, $S_{\mathrm{d}}(T)$, of a two-band conductor [49] as has been done previously for $\mathrm{UCoGa}_{5}$ [50] and $\mathrm{UCoGe}$ [51], for example, using the formula:

$$
S_{\mathrm{d}}(T)=\frac{\pi^{2} k_{\mathrm{B}}^{2} T}{3 e}\left\{\left[\frac{1}{N_{s}(\mathrm{E})} \frac{\mathrm{d} N_{\mathrm{s}}(E)}{\mathrm{d} E}\right]_{E=E_{\mathrm{F}}}-\left[\frac{1}{N_{p d f}(E)} \frac{\mathrm{d} N_{p d f}(E)}{\mathrm{d} E}\right]_{E=E_{\mathrm{F}}}\right\},
$$


where $N_{s}$ and $N_{p d f}$ denote our calculated sums of contributions to total DOS (displayed in figure 3), originating from all the conduction electrons, that is, $s$ and $p, d, f$, respectively. In this manner, we obtained the linear dependence of diffusion TEP with the $S_{\mathrm{d}} T^{-1}$ slope of $-0.0197 \mu \mathrm{V} \mathrm{K} \mathrm{K}^{-2}$, which is displayed in figure 13 (black dashed line). Assuming that $\xi=3$ in Eq. (13), we have obtained $E_{\mathrm{F}}=3.7$ $\mathrm{eV}$. As can be seen, the computed $S_{\mathrm{d}}(T)$ yields a negative linear temperature dependence, revealing a general trend of the experimental TEP at higher temperatures, due to dominating electron carriers. However, the slope of the estimated isotropic $S_{\mathrm{d}}(T)$ function is quite different from (smaller than) those of the experimental $S_{a}(T)$ and $S_{\mathrm{c}}(T)$ straight lines, which can be explained by strong directional anisotropy of the diffusion TEP, originating from the anisotropy of the FS having one of the sheets [electron-like one, displayed in figure 4(c)], being open in the $a$-axis but closed in the $c$-axis direction. A similar anisotropic behaviour was reported in Ref. [52] for orthorhombic compounds such as $\mathrm{ThCoGa}_{4}$, for which positive $S_{b, c}(T)$ functions are almost linear and have nearly the same slopes, while $S_{a}(T)$, which is also positive, is curvilinear and has much higher values than those for the other axes. Moreover, behaviour quite reminiscent of our $S(T)$ behaviour was found for the tetragonal system ThPS [53]. In the high-temperature range, the authors of this work also observed a nearly linear and negative slope of $S(T)$, whose intersection with the y axis at $T=0 \mathrm{~K}$ yields a negative small value as in our case. Thus, such a low-temperature deviation from the straight-line behaviour has been attributed to the presence of a phonon contribution, $S_{\mathrm{g}}(T) \sim T^{3}$.

\section{Summary}

We have studied the structural, thermal, magnetic, and electronic transport properties of $\mathrm{ThFe}_{2} \mathrm{Al}_{10}$ single crystals. We found that the specific heat data of this aluminide, obtained between 2 and $300 \mathrm{~K}$, consist of three components: Debye, Einstein, and electronic ones. We established the same contributions for the paramagnetic isostructural U-based aluminide. The specific heat curves for both ternaries exhibit anomalies at low temperatures, indicating the presence of spin fluctuations in the $\left[\mathrm{Fe}_{2} \mathrm{Al}_{10}\right]$ sublattice due to the closeness of this system to the quantum critical state, as observed previously for $\mathrm{YFe}_{2} \mathrm{Al}_{10}$. The electronic properties of $\mathrm{ThFe}_{2} \mathrm{Al}_{10}$ are typical of metals. Hence, the Th atoms, located in a very anisotropic cage, are weakly linked with the surrounding and are therefore able to undergo rattling vibrations at low temperatures. As we found from the magnetoresistivity measurements, such vibrations are distinctly observed only along the $c$ axis, where the thermal displacement factor $U_{\text {eq }}$ is therefore usually the highest (see Discussion in Ref. [54]). In the latter paper, the authors considered the $\mathrm{Ce}_{2} \mathrm{Al}_{10}$ systems showing the large anisotropy in the susceptibility which comes from the anisotropic exchange interaction. This is the largest along the $c$ axis. In our system, the large crystal field splitting, as is also the case of the Ce-systems, originates from strong anisotropic $c-f$ hybridization, being the strongest along the $a$ axis. Hence, any rattling in such anisotropic systems is possible predominantly along the $c$-axis. The first example of behaviour mentioned above was observed in the magnetoresistance of metallic $\mathrm{UB}_{12}$ [40], which belongs to cagetype compounds. An unveiling of such a phenomenon was possible only in the study of metallic caged uranium compounds due to an existing interaction between the rattling vibration of a low frequency $\left(\Theta_{\mathrm{E}} \approx 20 \mathrm{~K}\right)$ and the conduction band, as described in the literature. Furthermore, assuming that the transport behaviour of Th-based aluminide is of the phonon type, we used its resistivity data to separate the thermal behaviour of the resistivities in $\mathrm{UFe}_{2} \mathrm{Al}_{10}$, which are mainly connected with the scattering of electrons on crystal-field levels. The scheme of this level splitting of the ground multiplet was taken from our previous work [15]. We had to use also another contribution - a Kondo-like one to achieve a fit to the experimental data.

We also performed fully relativistic band structure calculations for the $\mathrm{ThFe}_{2} \mathrm{Al}_{10}$ reference compound without $5 f$-electrons. The results reveal its metallic-like character, with contributions coming from all the constituents' atoms, as in the case of $\mathrm{UFe}_{2} \mathrm{Al}_{10}$, and similarly large contributions of the Fe $3 d$ and $\mathrm{Al} 3 s p d$ electrons at the Fermi level. The difference between the FSs of $\operatorname{ThFe}_{2} \mathrm{~A}_{10}$ and $\mathrm{UFe}_{2} \mathrm{Al}_{10}$ appears because the former contains as many as four sheets of both hole-like and electronlike characters, resembling those in isostructural $\mathrm{CeRu}_{2} \mathrm{Al}_{10}$, whereas $\mathrm{UFe}_{2} \mathrm{Al}_{10}$ exhibits only two $\mathrm{FS}$ sheets. Such a difference is expected due to various types of chemical bonding between the central atom and its ligand cage. 


\section{Acknowledgments}

The Computing Centre at the Institute of Low Temperature and Structure Research of the Polish Academy of Sciences in Wrocław is acknowledged for use of the supercomputers and technical support.

\section{References}

[1] Gonçalves A P and Noël H 2005 Intermetallics 13580

[2] Kuznietz M, Gonçalves A P, Waerenborgh J C, Almeida M, Cardoso C, Cruz M-M and Godinho M 1999 Phys. Rev. B 609494

[3] Meshi L, Zenou V Y, Ezersky V, Munitz A and Talianker M 2002 J. Alloys Compd. 347178

[4] Zenou V Y, Meshi L and Fuks D 2011 Intermetallics 19713

[5] Meshi L, Talianker M and Munitz A 2004 J. Alloy Compd. 370206

[6] Noël H, Gonçalves A P and Waerenborgh J C 2004 Intermetallics 12189

[7] Halevy L, Zenou V Y, Salhov S, Caspi E N, Schäfer W and Yaar I 2006 J. Alloys Compd. 41921

[8] Thiede V M T, Ebel T and Jeitschko W 1998 J. Mater. Chem. 8125

[9] Muro Y, Motoya K, Saiga Y and Takabatake T 2009 J. Phys. Soc. Japan 78083707

[10] Kimura S, Iizuka T, Miyazaki H, Hajiri T, Matsunami M, Mori T, Irizawa A, Muro Y, Kajino J and Takabatake T 2011 Phys. Rev. B 84 165125, and references therein.

[11] Robert J, Damay F, Saito K, Bataille A M, Porcher F, André G, Gukasov A, Mignot J-M, Tanida H and Sera M 2014 Phys. Rev. B 90224425

[12] Strydom A M, Peratheepan P, Sarkar R, Baenitz M and Steglich F 2011 J. Phys. Soc. Jpn. 80 SA043

[13] Strydom A M, Khuntia P, Baenitz M, Paratheepan P and Steglich F 2013 Phys. Status Solid B 250630

[14] Sugai T, Haga Y, Matsuda T D, Yamamoto E, Tateiwa N, Honda F, Settai R and Ōnuki Y $2011 \mathrm{~J}$. Phys.: Conf. Ser. 273012122

[15] Troć R, Samsel-Czekała M, Talik E, Wawryk R, Gajek Z and Pasturel M 2015 Phys. Rev. B 92 104427

[16] Samsel-Czekała M, Talik E, Pasturel M and Troć R 2013 J. Alloys Compd. 554438

[17] Sheldrick G M 2001 SADABS, Bruker AXS Inc. Madison, Wisconsin, USA

[18] Altomare A, Burla M C, Camalli M, Cascarano G I, Giacowazzo C, Guagliardi A, Moliterni A G G, Polidori G, Spagna R 1999 SIR97 J. Appl. Cryst. 32115

[19] Sheldrick G M 2008 Acta Cryst. A 64112

[20] Parthé E, Cenzual E K, Gladyshevskii R 1993 J. Alloys Compd. 197291

[21] Wawryk R and Henkie Z 2001 Phil. Mag. B 81223

[22] Koepernik K and Eschrig H 1999 Phys. Rev. B 591743

(FPLO9.00-34; www.FPLO.de)

Eschrig H, Richter M and Opahle I 2004 Relativistic Electronic Structure Theory, Part 2. Applications (Theoretical and Computational Chemistry vol. 14) ed P. Schwerdtfeger (Amsterdam: Elsevier) p 723

[23] Perdew J P and Wang Y 1992 Phys. Rev. B 4513244

[24] Meshi L, Ezersky V, Venkert A and Talianker M 2005 Intermetallics 13792

[25] Perdew J P, Burke K and Ernzerhof M 1996 Phys. Rev. Lett. 773865

[26] Niemann S and Jeitschko W 1995 Z. Kristallogr. 210338

[27] Uziel A, Bram A I, Venkert A, Kiv A F, Fuks D and Meshi L 2015 J. Alloy Compd. 648353

[28] Teatum E, Gschneidner K, Waber J 1960 Compilation of calculated data useful in predicting metallurgical behavior of the elements in binary alloy systems, LA-2345 (Los Alamos Scientific Laboratory).

[29] Villars P, Cenzual K 2009 Pearson's Crystal Data: Crystal Structure Database for Inorganic Compounds, Release 2009/1 (ASM International, Materials Park, Ohio, USA) (and references therein)

[30] Matsuda T D, Haga Y, Yamamoto E, Ikeda S, Shishido H, Settai R, Harima H and Ōnuki Y 2007 J. Phys. Soc. Japan 76064712 
[31] Doniach S and Engelsberg S 1966 Phys. Rev. Lett. 17750

Berk N F and Schrieffer J R 1966 Phys. Rev. Lett. 17433

[32] Park K, Wu L S, Janssen Y, Kim M S, Marques C and Aronson M C 2011 Phys. Rev. B 84 094425

[33] Pecharsky V K, Hyun O B and Gschneidner Jr K A 1993 Phys. Rev. B 4711839

[34] Pecharsky V K and Gschneidner K A 1991 Phys. Rev. B 438238

[35] Wawryk R, Żogał O, Rudenko A, Cichorek T, Henkie Z and Maple M B 2016 J. Alloys Compd. 688478

[36] Khuntia P, Strydom A M, Wu L S, Aronson M C, Steglich F and Baenitz M 2012 Phys. Rev. B 86 220401(R)

[37] Cox D L and Zawadowski A 1998 Adv. Phys. 47599

[38] Cichorek T, Sanchez A, Gegenwart P, Weickert F, Wojakowski A, Henkie Z, Auffermann G, Paschen S, Kniep R and Steglich F 2005 Phys. Rev. Lett. 94236603

[39] Hanzawa K 2010 J. Phys. Soc. Japan 79084704

[40] Troć R, Wawryk R, Pikul A and Shitsevalova N 2015 Phil. Mag. B 952343

[41] Hiroi Z, Onosaka A, Okamoto Y, Yamaura J and Harima H 2012 J. Phys. Soc. Japan 81124707

[42] Gratz E and Markosyan A S 2001 J. Phys.: Condens. Matter 13 R385

[43] Rao V U S and Wallace W E 1970 Phys. Rev. 24613

[44] Durczewski K, Gajek Z and Mucha J 2014 Phys. Status Solidi B 2512265

[45] Hessel Andersen N and Vogt O 1979 J. de Physique 40 C5-118

[46] Frederick N A and Maple M B 2003 J. Phys.: Condens. Matter 154789

[47] Gajek Z et al (to be published)

[48] Bernard R D 1972 Thermoelectricity in metals and alloys (London: Taylor and Frabcis LTD)

[49] Blatt F J, Schroeder P A, Foiles C L and Greig D 1976 Thermoelectric Power of Metals (New York: Plenum) p 147 (and references therein)

[50] Troć R, Bukowski Z, Sułkowski C, Misiorek H, Morkowski J A, Szajek A and Chełkowska G 2004 Phys. Rev. B 70184443

[51] Troć R, Wawryk R, Miiller W, Misiorek H and Samsel-Czekała M 2010 Phil. Mag. 902249

[52] Wawryk R, Stępień-Damm J, Henkie Z, Cichorek T and Steglich F 2004 J. Phys.: Condens. Matter. 165427

[53] Wawryk R, Wojakowski A, Pietraszko A and Henkie Z 2005 Solid State Commun. 133295

[54] Tanida H, Tanaka D, Sera M, Tanimoto S, Nishioka T, Matsumura M, Ogawa M, Moriyoshi C, Kuroiwa Y, Kim J E, Tsuji N and Takata M 2011 Phys. Rev. B 84115128 
* Low-frequency Einstein vibrations (rattling) of Th atom detected in $\mathrm{ThFe}_{2} \mathrm{Al}_{10}$.

* Spin fluctuations on $\mathrm{Fe}$ atoms observed in the above Th-based system.

* Proximity to quantum critical point concluded for $\mathrm{ThFe}_{2} \mathrm{Al}_{10}$.

* For the first time crystal field effects analyzed in resistivity of actinides. 